\section{EMBRYRIDDLE \\ Aeronautical University}

SCHOLARLY COMMONS

\section{International Journal of Aviation,} Aeronautics, and Aerospace

7-18-2016

\title{
Optimization of Takeoffs on Unbalanced Fields using Takeoff Performance Tool
}

Nihad E. Daidzic

AAR Aerospace Consulting, LLC, aaraerospace@cs.com

Follow this and additional works at: https://commons.erau.edu/ijaaa

Part of the Aerospace Engineering Commons, Applied Mathematics Commons, Aviation Commons, Dynamics and Dynamical Systems Commons, and the Engineering Mechanics Commons

\section{Scholarly Commons Citation}

Daidzic, N. E. (2016). Optimization of Takeoffs on Unbalanced Fields using Takeoff Performance Tool. International Journal of Aviation, Aeronautics, and Aerospace, 3(3). https://doi.org/10.15394/ ijaaa.2016.1129

This Article is brought to you for free and open access by the Journals at Scholarly Commons. It has been accepted for inclusion in International Journal of Aviation, Aeronautics, and Aerospace by an authorized administrator of Scholarly Commons. For more information, please contact commons@erau.edu. 
Conventional airplane horizontal takeoff run consists of the accelerating ground-roll phase and the airborne phase. The airborne phase starts at liftoff with $v_{L O F} \geq v_{M U}$ to regulatory screen height $(\mathrm{SH})$ at $v_{S H} \geq v_{2}$. Part of the airborne takeoff run does not require hard-surface underneath, but obstacle-free space instead. Hence, clearways (CWY) are often used to facilitate part of the airborne takeoff segment and accordingly increase operating takeoff weight (TOW). The entire takeoff maneuver is considered accomplished by reaching 1,500 ft AGL at flapsup maneuvering speed (Holt and Poynor, 2006; Swatton, 2008). Takeoff regulations of transport-category (T-category) airplanes with associated speed requirements are covered in respective FAR/CS 25.105, 25.107, 25.111, 25.113 and 25.115 (EASA, 2007; FAA, 2011, 2013). Scheduled takeoff performance must satisfy numerous constraints imposed by FAR/CS 25 and some operational rules, such as, 91.605, 121.189 (FAA, 2014b), and 135.379. Stopways (SWYs) and CWYs were introduced in the early 1950's by the ICAO (International Civil Aviation Organization) and the British Civil Aviation Authorities (CAA) in order to accommodate the operation of the first commercial jet transport - Comet 1 (Smith M. A., 1953).

In most engineering references, a so-called, direct takeoff-dynamics problem is solved. The weight (mass) of an airplane is known, while the aerodynamic, propulsive, gravitational, and frictional forces and accelerations are modeled. Most of the takeoff occurs in ground effect and at constant pitch attitude. The required distance for takeoff is then calculated by marching-in-time integration of differential equations of motion based on speed-dependent forces. However, airplane takeoff problems in scheduled air-carrier operations are more complex and the solution of the more difficult inverse problem is sought. Takeoff runways are now fixed. Existing environmental conditions must be used over which an operator has no control. The maximum takeoff weight at available thrust and discrete flap setting that can be lifted is maximized under many constraints. Thus, the problem of T-category takeoffs is a problem of nonlinear programing (Luenberger, 1984; Minoux, 1986; Pierre, 1984). The constrained nonlinear optimization of the system of algebraic equations and inequalities is solved. It is not possible to solve this problem in a closed (analytical) form unless some severe, and totally unrealistic, assumptions are made.

Runways used for T-category commercial jets are very expensive ( $\$ 600$ $\$ 1,000 / \mathrm{ft}^{2}$ ). An airport operator can opt for a cheaper option and that is to add SWY to increase accelerate-stop distance available (ASDA) and CWY to increase takeoff distance available (TODA). General runway layout is illustrated in Figure 1. Note that EMAS cannot be used in takeoff calculations. Adding CWY only will increase field-length limited takeoff weight (FLLTOW), but at the cost of lower 
decision/action speed $V_{l}$ to account for unchanged ASDA and heavier weight. Complete regulatory definitions of CWYs and SWYs is provided in 14 CFR 1, FAA (2014a), ICAO (2006), Swatton (2008), and will thus not be repeated. For runways with no CWYs, TODA is more restrictive than takeoff run available (TORA). TORA provides full-strength pavement surface capable of supporting given airplane operations during all field operations. Regulations allow maximum usable CWY to be no longer than 50\% of TORA. However, as the CWY is added to the basic TORA, the takeoff run required (TORR) becomes significant and at a certain CWY length, takeoff distance required (TODR) and TORR are equally restrictive. The CWY for which FLLTOW TODR $=$ FLLTOW $_{\text {TORR }}$ is designated critical $C W Y$. Any CWY beyond critical is practically unusable. Accelerate-stop (AS) and accelerate-go (AG) T-category airplane takeoff scenarios, available and required distances are illustrated in Figure 2.

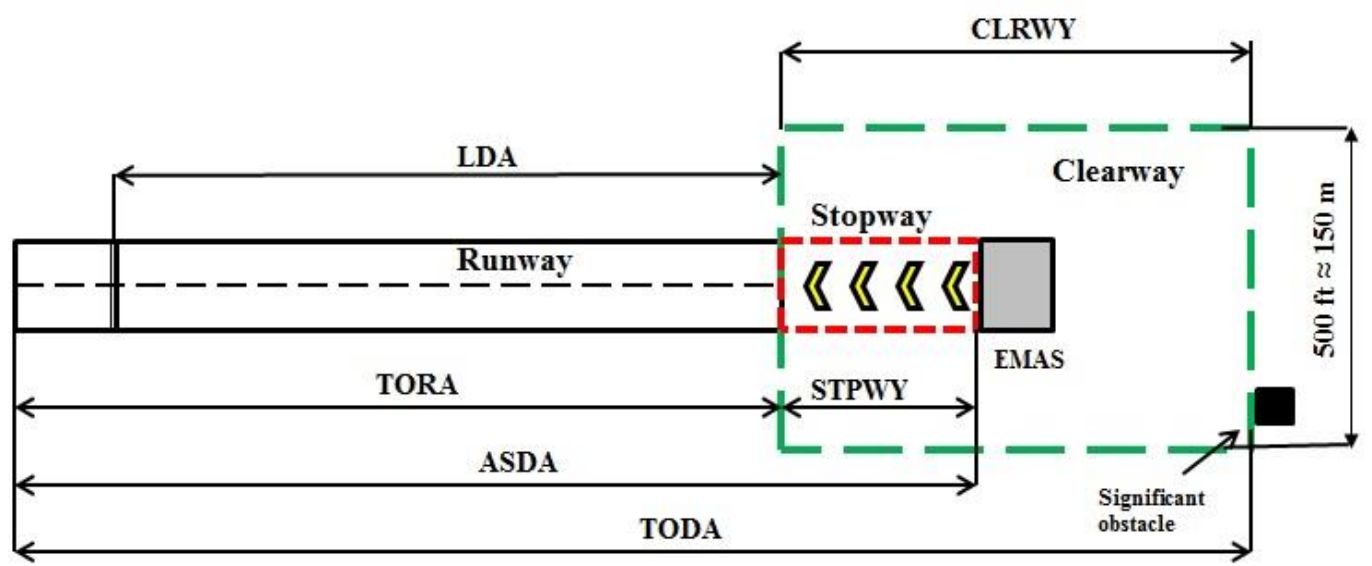

Figure 1. General runway layout. Not to scale.

By definition, the balanced field length (BFL) represents the case for which ASDA=TODA. On the other hand, the balanced field condition (BFC) occurs when ASD=TOD (or ASDR=TODR). One may have BFL, but not BFC due to the effect of runway slope, wind, density altitude (DA), etc. Similarly, the field lengths may be unbalanced (UBFL), but the BFC may still exist. Inconsistent definitions and use of BFL and BFC is common in practice. In this work, we will primarily focus on UBFL and typically in real life that implies TODA > ASDA. Although the field may be unbalanced, a unique $V_{l}$ and FLLTOW may exist. A symbolic depiction of BFL and UBFL is shown in Figure 3. Hence, $V_{l}$-speed represents a pitchfork bifurcation point after which two entirely different takeoff histories exist. There are infinitely many possible combinations of SWYs, CWYs and TORAs. Sometimes starter extensions (up to $500 \mathrm{ft}$ long) are used to increase TORA (Swatton, 2008). BRP defines brake release point. 


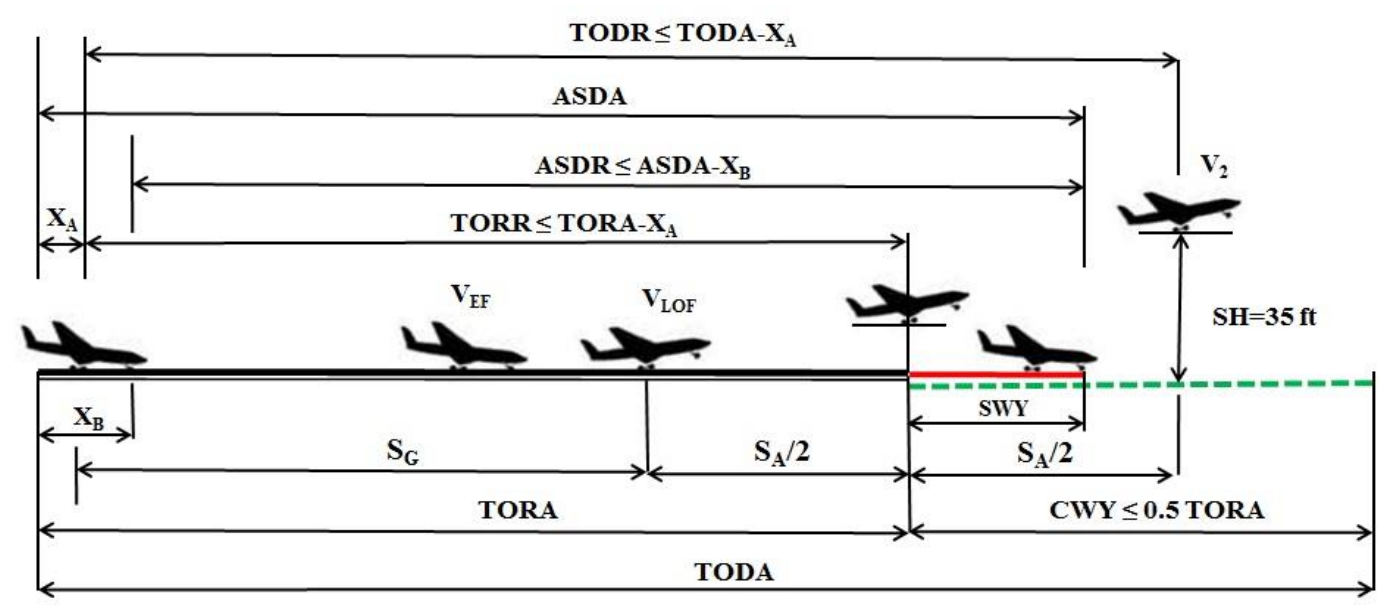

Figure 2. Available and required takeoff distances for a typical unbalanced condition. Not to scale.
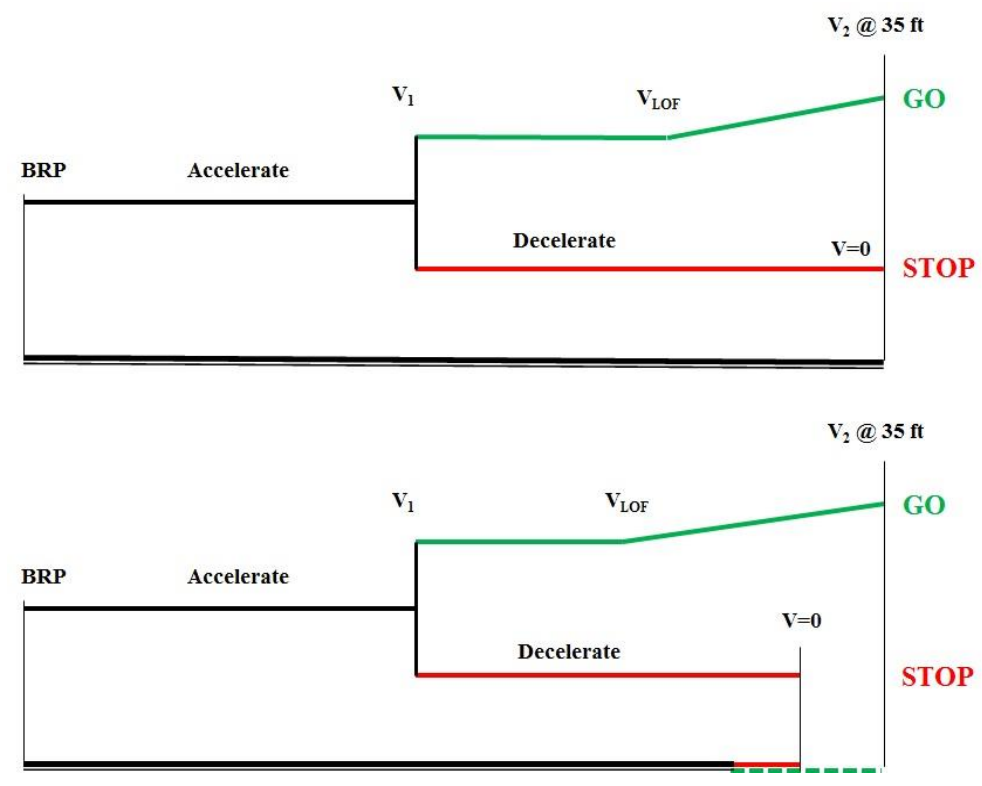

Figure 3. Symbolic depiction of balanced (above) and arbitrary unbalanced (below) takeoffs from dry, level, and paved runways. Not to scale.

Certified TOWs are obtained by flight-tests in prototype airplanes followed up by data reduction to standard conditions. Normally, any of the following conditions can limit regulated TOW (RTOW): TORA, ASDA, or TODA. RTOW is the lower of the maximum structural TOW (MSTOW) and the performance-limited TOW (PLTOW). This can be evaluated for net (factored) all- 
engines-operating (AEO) and gross (unfactored) one-engine-inoperative (OEI) cases for dry or wet conditions. Additional limitations can be posed by the maximum brake energy absorption, which limits the maximum groundspeed for AS rejected takeoff (RTO), $v_{1} \leq v_{M B E}$. On the other hand, the maximum liftoff groundspeed $v_{\text {LOF }} \leq v_{\text {TIRE }}$, limits AG maneuver. The takeoff decision/action speed $V_{l}$ is aerodynamically limited by $v_{M C G}$ on the lower end $\left(v_{M C G} \leq v_{E F}\right)$, and rotation $v_{R}$, on the higher-end of the speed-spectrum. Physically, ASDA defines the envelope of the $V_{S T O P}$ or maximum $V_{l}$ speeds, while TODA/TORA defines the envelope of the minimum $V_{l}$ or $V_{G O}$ speeds, such that, $v_{G O} \leq v_{1} \leq v_{S T O P}$. AEO TORR and TODR do not depend on $V_{l}$. Definitions and details of various takeoff $V$ speeds can be found in Airbus (2002), Swatton (2008), and current FAR 25.51.

Physics of AS and AG maneuvers is complex. Although, the laws of the classical (Newtonian-Lagrangian-Hamiltonian) physics/mechanics are well known and straightforward to apply in differential or integral form, accounting for, and estimating, all aerodynamic, propulsive, mechanical, gravitational, and other forces (and moments) acting on accelerating aircraft is an extremely arduous task plagued with uncertainties. Although not inertial, non-rotating flat-Earth topocentric frame of reference fixed to the airfield was used with takeoff equations of motion. Energy balances during takeoff are illustrated in Figure 4.

The FAA Amendment 25-92 and guide to flight testing of FAR 25 airplanes summarized in AC 25-7C (FAA, 2012) defines how are certification rules to be applied in order to arrive to figures contained in airplane flight manuals (AFM). For FAR 25 airplane certification process, multiple experimental and fully instrumented airplane prototype demonstrated takeoff runs are required (Daidzic, 2013b), resulting in average values scaled/reduced to appropriate ISA conditions, configurations, and weights. Such is, of course, complex and expensive endeavor and currently the only option to certify field performance FAR/CS 25 airplanes. Alternatively, one can design 3-DOF or 6-DOF (or even $\infty$ DOF elastic airplane) simulation models consisting literary of hundreds of nonlinear ordinary differential equations (ODE) and simultaneous algebraic equations. Such initial-value ODE systems can be then solved using sophisticated numerical integration methods, such as, adaptive single-step Runge-Kutta, Richardson extrapolation and the Burlish-Stoer methods, multi-step AdamsBashforth-Moulton methods, and predictor-corrector methods as sub-category of multi-step methods (Allerton, 2009; Chapra and Canale, 2006; Press et al., 1992). Such complex nonlinear simulation models would surpass the fidelity used in current Level-D full flight simulators, cannot run in real time (Allerton, 2009), and provide little insights into dynamics of field performance. Due to convoluted 
simultaneous effects of many parameters it is difficult to draw definite conclusions or learn much from it. Parametric analysis is possible, but it requires hundreds of hours of tedious work and in case of flight tests would be practically impossible to conduct. Flight test results supported by physics-based data reduction are then entered in AFMs.

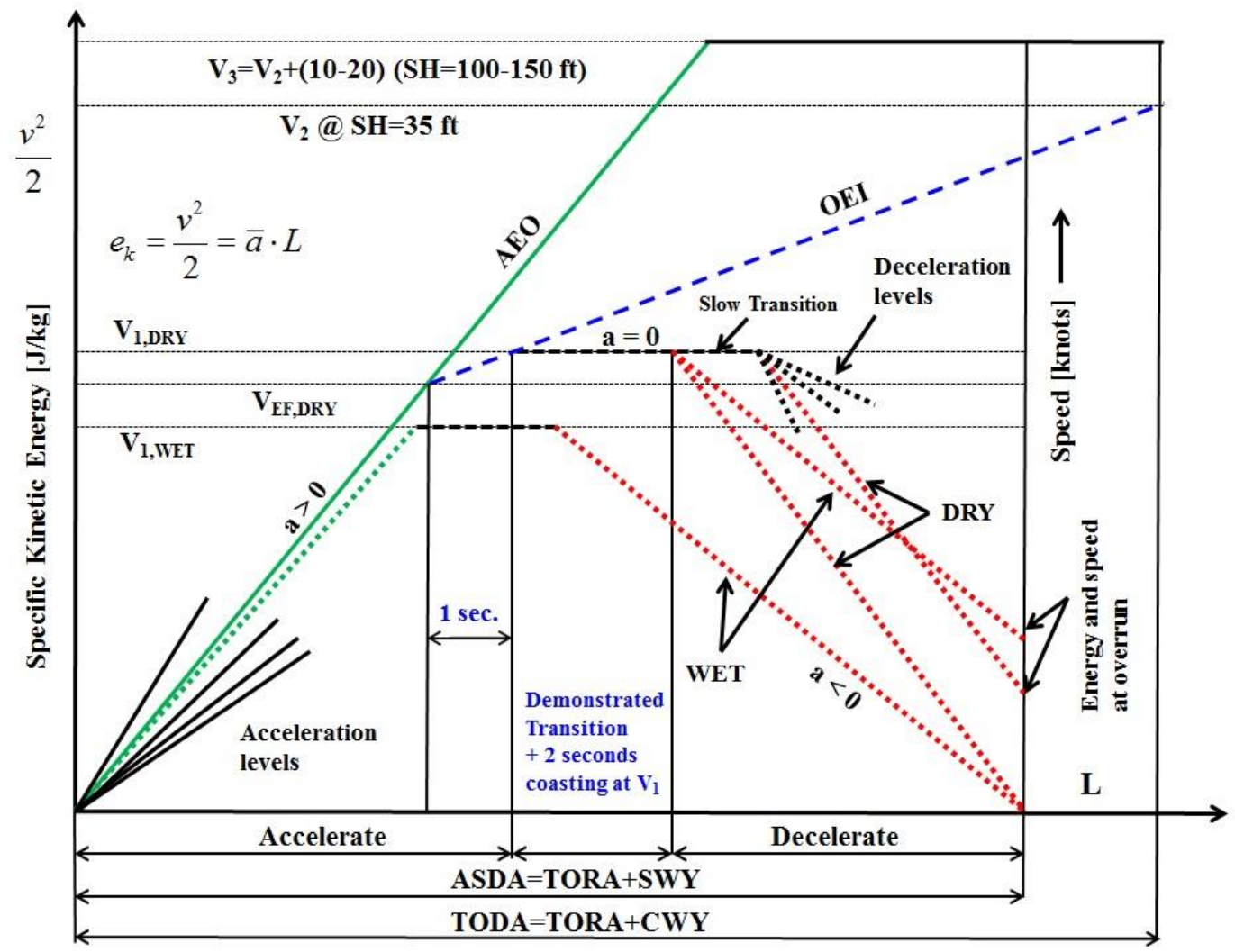

Figure 4. Illustration of energy balances during specific unbalanced AEO and OEI AG and AS takeoff. Not to scale.

A total-energy physics-based model of FAR/CS 25 airplane takeoff performance was developed here. This model uses existing certification and operational regulations, but is flexible and has many free parameters that can be adjusted to simulate any regulatory changes, runway data, environmental data, and particular airplane/engine models. The model operates by utilizing the energy conservation principle on integral (macroscopic) scale. As such it cannot recreate takeoff time histories, but the end states only. While, real-world aircraft takeoff performance certification cannot be currently based on mathematical models, it is hoped that simulated results will be very close to demonstrated performance. 
Thus, the main focus of our study is to build a realistic, yet simple enough, mathematical model of FAR/CS 25 certified airplanes takeoff maneuver complying with all applicable regulatory, environmental, runway, and aircraft constraints/limitations. This mathematical model and simulation software was named Takeoff Performance Tool (TPT). The presented physics-based totalenergy model serves several purposes. It provides deeper insights into takeoff physics and highlights the relative importance of various regulatory, aircraft, runway, environmental and atmospheric parameters. Hence, TPT can be used in analytical and numerical takeoff optimization studies, airport/runway planning and operations, economic analysis of runways, CWYs, and/or SWYs, etc. Another goal was to evaluate how unbalanced fields affect TOW and $V_{l}$.

\section{Literature Review}

Not much information on T-category airplane takeoff performance theory and applications in commercial service is available in public domain. It is assumed and expected that large civil-aircraft manufacturers, such as, Airbus, Boeing, Embraer, etc., would have developed proprietary takeoff performance models and programs augmented by flight test data (i.e., flight-test expansion models). For example, Airbus (2002) provides useful information and performance analysis during various phases of flight for its customers. Airbus has developed standalone proprietary PEP (Performance Engineering Programs) application in MS Windows environment designed to provide the necessary tools to handle the performance aspects of flight preparation and also to analyze aircraft performance after the flight. PEP consists of many submodules specializing in aircraft performance calculations for various phases of flight (Airbus, 2002). For example, OCTOPUS (Operational and Certified TakeOff and landing Performance Universal Software) is used for field performance computations related to A320 family, A330, A340, and A380. It was designed in Fortran 95 by Airbus with roughly 230,000 lines of code (de Lemos Viana, 2011). More details on OCTOPUS applications can be found in de Lemos Viana (2011). Details on internal workings and optimization algorithms are not available.

Takeoff dynamics of rigid airplanes is described in various levels of detail in several standard and classical aerospace/aeronautics engineering references, such as, Anderson (1999), Asselin, (1997), Eshelby (2000), Filippone (2012), Hale (1984), Mair and Birdsall (1992), McCormick (1995), Ojha (1995), Padilla (1996), Roskam and Lan (1997), and Vinh (1993). However, all these references deal with the direct takeoff problem, i.e., by knowing airplane's TOW find the required takeoff distances for given runway and environmental conditions. The problem facing commercial operators (FAA, 2014b) daily is actually the inverse 
takeoff problem, i.e., knowing the available/declared takeoff distances (ASDA, TORA, TODA), find the maximum operating or restricted TOW under known environmental and runway conditions while complying with several constraints. No general analytical solution of such nonlinear inverse problem exists.

From the flight testing, piloting, and airline operational perspective, we reference the work of Davis (1971), Lowery (2001), Webb and Walker (2004) and Holt and Poynor (2006). Davis (1971) gives a good early account of certification rules and flight tests on B747-100. Lowery (2001), Web and Walker (2004) and Holt and Poynor (2006) give account of many takeoff operational regulations as well as takeoff piloting techniques and procedures. FAA's takeoff training safety aid (FAA, 1994), gives detailed qualitative account of many RTO accidents and highlights takeoff techniques and procedures in non-mathematical terms. Also, many incidents and accidents have occurred in airfield line operations (during takeoff and landing phase) due to erroneous input of performance data (ATSB, 2011). Some of the accidents are summarized in ATSB (2011), but there is no space here to discuss or go into detail on any of the particular events.

A good early source of flight testing procedures and data reduction is given in Durbin and Perkins (eds.) (1962). Takeoff performance measurements and reduction to standard conditions is properly described. Perry (1967) gives an overview of fixed-wing aircraft horizontal normal-takeoff physics highlighting many major factors. The author showed how to solve the direct-takeoff problem using both the energy- and the differential-models. It is often possible to analytically integrate differential models to arrive at takeoff distance-required solutions in a closed form implying some, more or less severe, assumptions must be first made. Wagenmakers (1991) discuss many topics of interest regarding aircraft performance monitoring, regulations, and planning. The author defines in clear terms the needs for better understanding of some takeoff issues. Roskam and co-workers developed Advanced Aircraft Analysis (AAA) modules and much of the underlying implemented theory can be found in Roskam and Lan (1997). Nuic et al. (2005) present advanced aircraft performance modelling tool called BADA (Base of Aircraft Data). The primary purpose of BADA is to serve as a tool in air traffic management. BADA model is based on kinetic (dynamic) physics-based first-principle differential models that account for various forces. Integration of the underlying system of differential equations describing forces and the totalenergy state of aircraft at every instant results in variable-mass (weight) trajectories in time and space for all phases of flight. Aircraft limitations are also implemented. Large internal aircraft database is required to provide inputs into the model. Similarly, a powerful PIANO software (Simos, 2006) was designed as a performance analysis tool, aircraft database and preliminary design tool. This 
proprietary commercial software has almost become an industry standard with many prominent users, but not much information and details of its internal working is available in public domain

ICAO's aerodrome design manual (ICAO, 2006) gives very good description of the effect that SWYs and CWYs have on the takeoff performance. In general, very little to none qualitative information was found on the effect of SWYs and CWYs on takeoff performance. In an aviation-industry oriented article Chiles (2007) discusses some takeoff myths and common practices and highlights the need for takeoff performance monitoring. Jeppesen (2007) and Swatton (2008) give account on various takeoff regulations and operational procedures in FAR/CS 25 airplanes mostly aimed toward professional pilots and commercial operators. Daidzic and Shrestha (2008) studied the FAR/CS 25 landing dynamics of T-category airplanes. The 3-DOF differential equations of motion model included realistic simulation of pilot actions and operational delays when landing on contaminated runways in adverse weather conditions. The model could also be used for RTO simulations, as a landing calculator, and as a tool in aircraft accident investigations. The level of sophistication in accounting for various runway contaminants surpasses the one used in Level-D Flight simulators. Filippone (2008) presents multi-disciplinary comprehensive analysis of $\mathrm{T}$ category aircraft flight performance. The author uses B777-300 with GE-90 engines as an example for performance calculations. Type of results obtained by performance analysis includes air-range charts, takeoff WAT charts, payloadrange performance, economy Mach number, etc. Ohme (2009) reports on a new developed software tool, called MAPET II for evaluation of aircraft takeoff and landing performance based on 6-DOF high-fidelity model. Ohme's model was designed for FAR/CS 25 airplane takeoff and landing dynamics where flap setting, CG location, runway contaminants and other parameters could be set through graphical user interface. A summary of some industry and academic aircraft performance programs is provided in Filippone (2012). The author also presents his differential model of takeoff performance. It is essentially a lumpedparameter model consisting of eight coupled nonlinear ODE. The author uses fixed (not adaptive) single-step Runge-Kutta methods to perform integration in time. While time histories can be recreated, this model does not solve the inverse takeoff problem nor does it perform optimization of TOW for given runway under given constraints. The same arguments can be used for all other previous works mentioned here. Torenbeek (2013) provides a wealth of theory and details on optimization of aircraft design. The takeoff maneuver was only marginally discussed and the statement was made that it is the most difficult of all the optimization studies. No constrained optimization and maximization of the Figure of Merit (FOM) of takeoff maneuver was presented. Zontul (2013) presents study 
in which all aircraft types can be combined in a single server based database systems. Author claims that its rule checking process is very dynamic and flexible and that server database can be extended to create national or international aircraft and airport information system in the future. Daidzic (2014a) presents analytical total-energy model and solutions of improved or overspeed- $V_{2}$ airplane takeoffs. Such takeoffs are often an important tool to increase TOW (and profit) when dealing with climb-limited takeoffs (CLTOW). Bays and Halpin (2014) propose replacements of traditional AFMs with the direct performance calculations from physics-based models. The results of their study shows that inherent conservatism in traditional AFMs causes undue penalty in airfield performance. Furthermore, sometimes even that conservatism is inadequate. Wasiuk et al. (2015) developed and used the Aircraft Performance Model Implementation (APMI) software to calculate the global commercial aviation fuel burn and emissions. Daidzic (2016a) and Daidzic (2016d), introduces and describes certain aspects of the proprietary Total Runway Safety System (TRSS) which falls under the Aircraft-Runway Total Energy Monitoring and Management Systems (ARTEMS). TRSS monitors, manages, and controls longitudinal and lateral trajectories of conventional fixedwing airplanes during field operations preventing overruns and veer-offs, including during RTO's.

\section{Mathematical Models and Methodology}

The first-principle physics-based aircraft performance models require knowledge and accounting of all dominant forces at every instant. That also requires in-depth knowledge of aircraft aerodynamics, structural, and dynamics data that sometimes even the manufacturer does not have readily available. Airplane takeoff is accelerated maneuver complicated by the fact that it involves three distinct stages occurring in changing ground effect magnitude:

1. Ground run (roll) acceleration (groundspeed 0 to airspeed $V_{R}$ ).

2. Rotation (airspeed $\mathrm{V}_{\mathrm{R}}$ to $\mathrm{V}_{\mathrm{LOF}}$ ) and transition to flight.

3. Accelerated climb to $\mathrm{SH}$ (airspeed $\mathrm{V}_{\mathrm{LOF}}$ to $\mathrm{V}_{2}$ @ OEI or $\mathrm{V}_{3}$ @ AEO).

Each of these phases involves multiple truly complicated interactions between an airplane, runway, and the environment. The TPT model derived here consists of four submodules: (1) Runway model (dry, wet, contamination, average slope, etc.), (2) Aircraft model (drag polars for various configurations, brakes, tires, etc.), (3) Propulsion engine model (thrust, SFC, anti-ice on/off, AC on/off, etc.), and (4) Environmental/atmospheric model (air density, wind, etc.). 
A total-energy based, model of airplane takeoffs is used in this study (Daidzic, 2014a, 2016b). Topocentric flat and non-rotating Earth, nearly inertial, frame-of-reference fixed at the runway threshold is used in derivation of the model equations. Horizontal distance covered during the constant net average acceleration or deceleration only depends on the groundspeeds at the beginning and at the end of the speed-range:

$\Delta s_{c-d}=\int_{v_{c}}^{v_{d}} \frac{v d v}{a}=\frac{v_{d, G S}^{2}-v_{c, G S}^{2}}{2 a}>0$

where, $v_{G S}=v_{T A S}-v_{w}=\frac{v_{E A S}}{\sqrt{\sigma}}-v_{w}$ and $\quad v_{T A S}=\frac{v_{E A S}}{\sqrt{\sigma}}$

Equation (1) describes kinematic problem. If $a<0$ (deceleration or negative acceleration), then $V_{c}>V_{d}$, and the distance covered is positive (deceleration length). All subsequent analysis assumes no-wind situation and thus $V_{G S}=V_{T A S}$. Since the equivalent airspeeds (EAS) are mostly used as reference airspeeds, we convert true airspeeds (TAS) to equivalent EAS, yielding:

$\Delta s_{c-d}=\int_{v_{c}}^{v_{d}} \frac{v d v}{a}=\frac{v_{d, E A S}^{2}-v_{c, E A S}^{2}}{2 \sigma a}>0$

The difference between CAS and EAS during takeoffs is less than one knot (for Mach < 0.3 and altitude < 10,000 ft) and thus negligible (Asselin, 1997; Eshelby, 2000; Hurt, 1965; Padilla, 1996). Dry air properties are calculated according to ISA model (Daidzic, 2015a, 2015b):

$$
\sigma=\frac{\rho}{\rho_{S L}}=\frac{\delta}{\theta}, \quad \delta=\frac{p}{p_{S L}}, \quad \theta=\frac{T}{T_{S L}}, \quad T_{S L}=288.15 \mathrm{~K}, \quad p_{S L}=101,325 \mathrm{~Pa}
$$

Model of standard troposphere was used to calculate air parameters (Daidzic, 2015a). It is thus assumed that all speeds are EAS from now on unless explicitly defined otherwise. From Equation (2), the Law of conservation of kinetic energy describes dynamic problem:

$$
\Delta s_{c-d}=\frac{W \cdot\left(v_{d}^{2}-v_{c}^{2}\right)}{2 \sigma g} /\left(\sum_{i} \bar{F}_{i}\right)
$$


Forces acting on airplane during takeoff are generally speed-dependent. Changing ground effect plays especially important role during airborne segment. An illustration of various forces acting on an airplane taking off are depicted in Figure 5. Modeling of average forces is summarized in Appendix A.

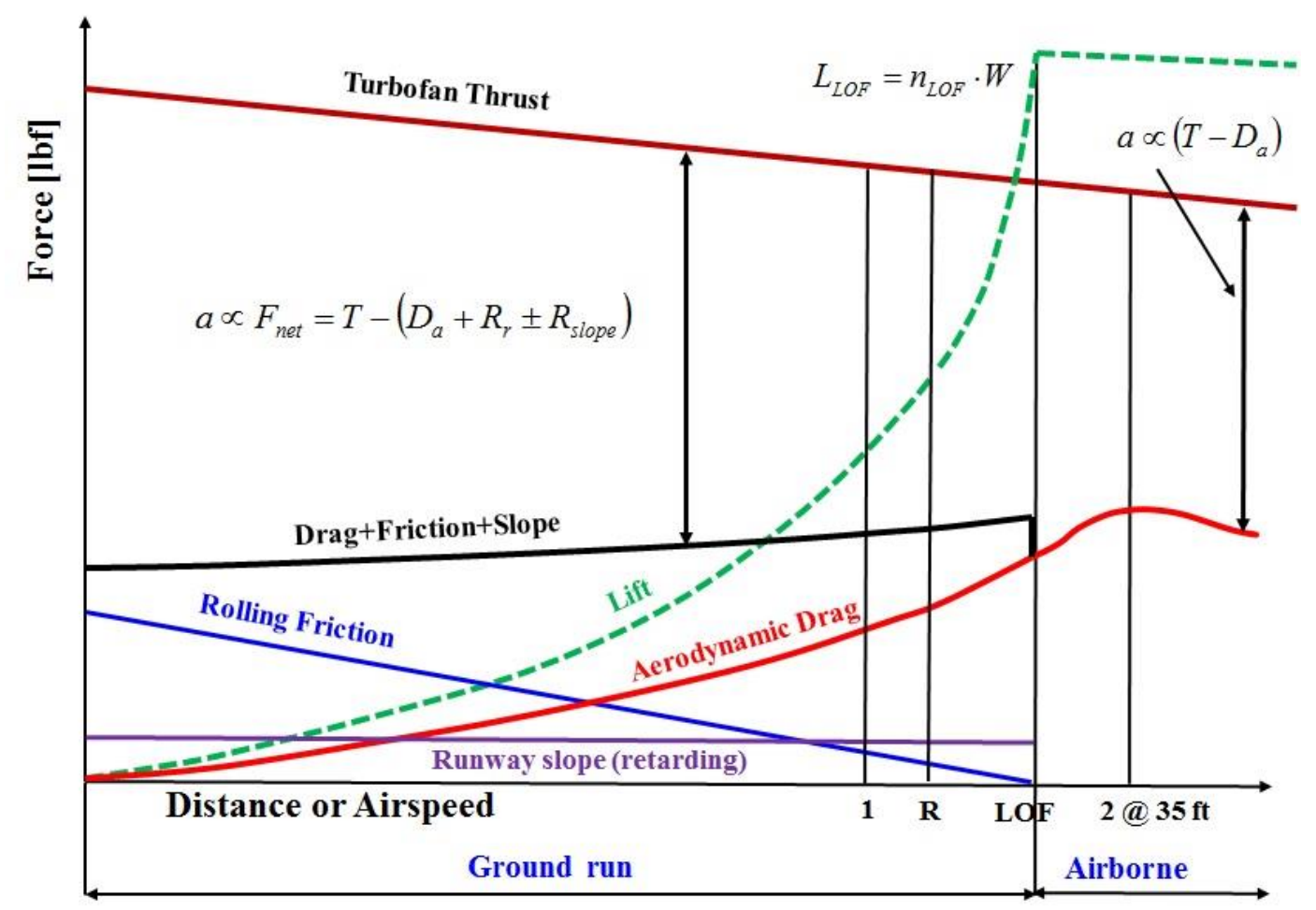

Figure 5. Main forces acting on accelerating airplane during one-dimensional along-the-runway force takeoff analysis. Not to scale.

Rolling resistance, in the absence of impingement and slush drag, is really a minor force especially as the effective weight on wheels decreases with speed. The rolling coefficient of friction (mostly adhesion and some hysteresis) on dry paved asphalt/concrete runways is about $0.010-0.025$ for most aviation tires (radial ply, bias ply) and is inflation pressure (filled mostly with $\mathrm{N}_{2}$ ) dependent. Visco-elastic rubber material is not necessarily following the Coulomb's law-offriction (e.g., Formula 1 racecars). The tire's coefficient of rolling and braking friction is also longitudinal-slip and speed dependent. Additional dependence comes from the runway surface (dry, damp, wet, contaminated). These facts are illustrated in Figure 6 for braking coefficient of friction as a function of surface quality and tire slip for a given speed. 


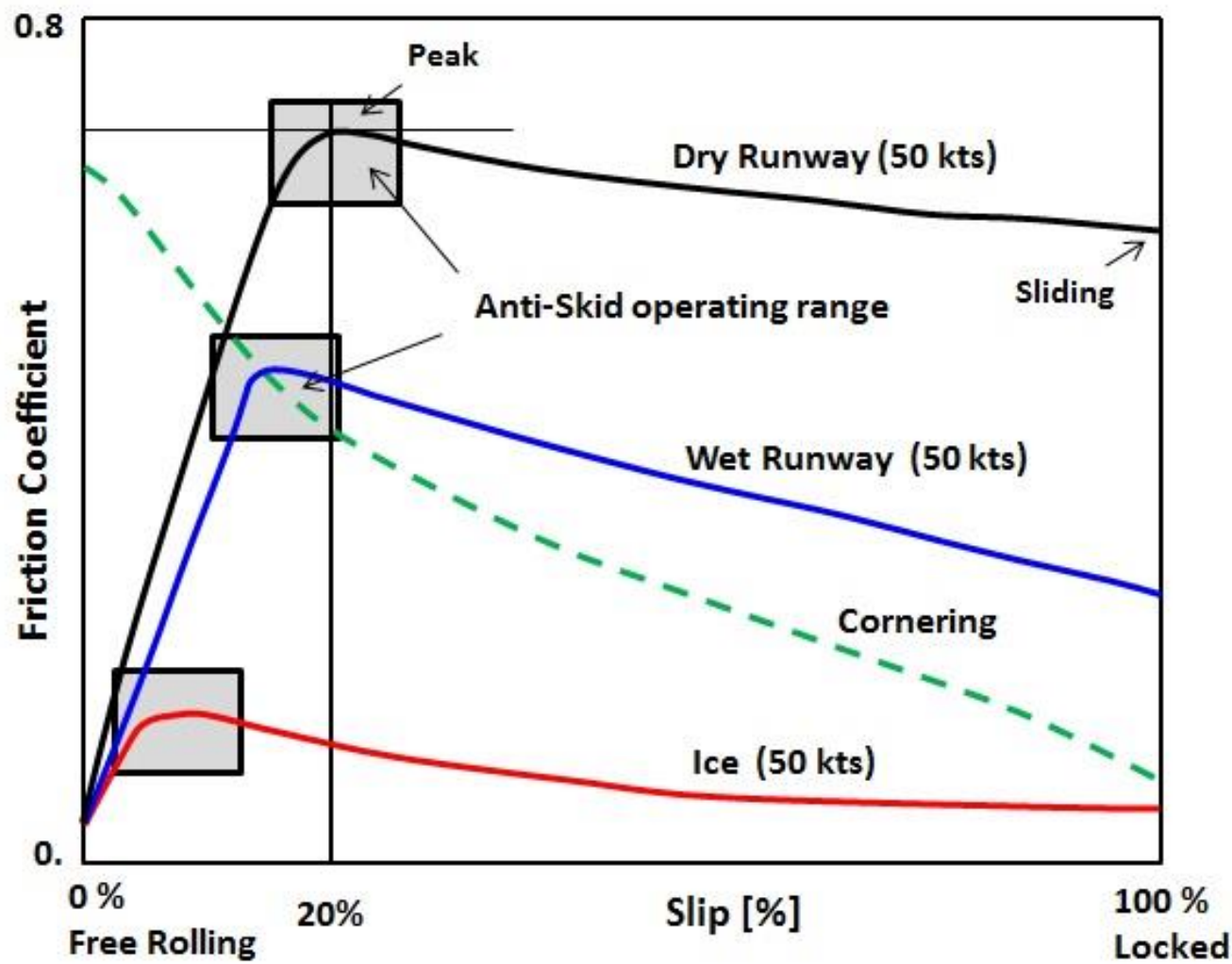

Figure 6. The coefficient of braking friction for various surfaces as a function of tire longitudinal slip and with anti-skid operating range depicted. Not to scale.

The speed-dependent peak braking coefficient of friction with the fullymodulated anti-skid system, for dry and wet level paved runways as a function of groundspeed are illustrated in Figure 7. Asselin (1997) reports peak braking coefficient of about 0.5, while Mair and Birdsall (1992) show linear dependence of braking coefficient with groundspeed with average dry concrete runway braking coefficient of 0.55 . Daidzic and Shrestha (2008) model the braking coefficient using linear speed dependence for various runway surface conditions. Durbin and Perkins (1962) show that for dry paved runway at groundspeed of 140 knots, the braking coefficient can be as low as 0.25 . The runway slope may and normally does change (positive or negative slopes) along the runway length and is thus a function of longitudinal distance. See Appendix A for more details on modeling of rolling and braking friction forces. The average aircraft stopping deceleration and force can be defined as:

$\bar{a}_{S T P}=\frac{g}{W} \cdot \bar{F}_{S T P}=\frac{g}{W} \cdot\left[\varsigma_{R E V} n_{e} \bar{T}_{1 e}-\bar{D}_{a}-\bar{\mu}_{B}(W-\bar{L})-\bar{\phi} W\right]=\mu_{S T P} \cdot g$ 


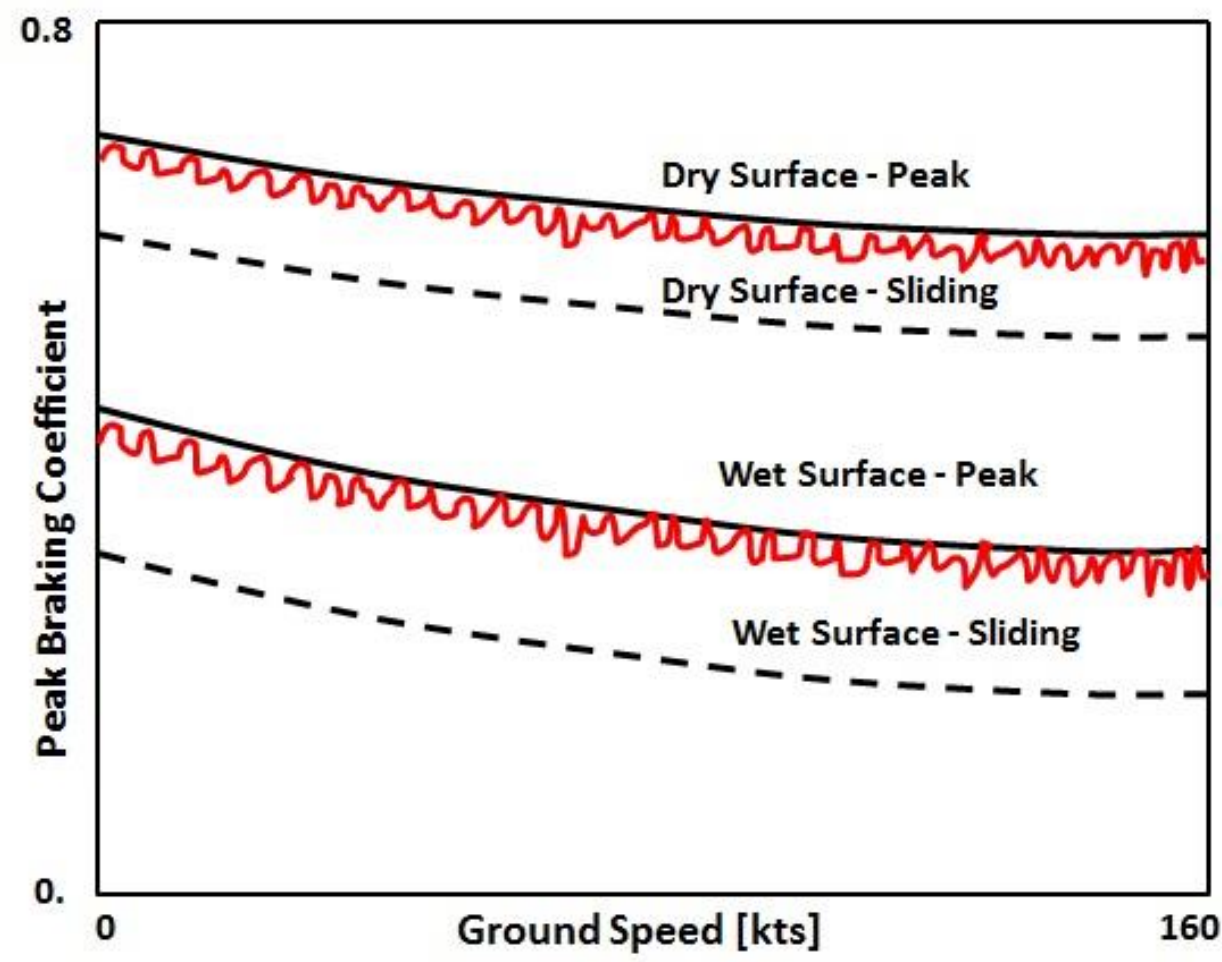

Figure 7. Peak coefficient of braking friction as a function of groundspeed for dry and wet surfaces and with the fully-modulated anti-skid operations. Not to scale.

The reverse-thrust coefficient $\varsigma_{R E V}$ could take any positive value between, typically, 0.07-0.1 (idle forward residual thrust) or about negative 0.3 to 0.45 for maximum AEO reverse-thrust in modern turbofans. The number of engines factor $n_{e}$ defines AEO and OEI cases. The amount of reverse thrust will of course depend on the engine make $\&$ model. Thrust reversers are allowed only for wetrunway certification. If the runway slope effective gradient is positive (upward), then it will assist stopping, if negative (downward) then the angle will be negative and provide gravity-assist thrust. The average friction braking force is very complicated and will also depend on the efficiency of the anti-skid system, braking coefficient of friction (tire/runway contact), average net weight on the tires (function of spoilers deflection and efficiency), etc. (Daidzic, 2011):

$$
R_{B}=\left(\bar{\mu}_{b} \cdot \eta_{\text {Beffort }} \cdot \eta_{A B S} \cdot \eta_{\text {Time }} \cdot \eta_{\text {Weff }}\right) \cdot W=\mu_{B} \cdot W \quad \eta_{\text {Weff }}=\overline{\left(1-\frac{L}{W}\right)}
$$

Nose gear typically supports about $10 \%$ of the entire aircraft weight and, in most cases, is not contributing to friction braking (Daidzic and Shrestha, 2008). 
Dynamic braking on main gears will cause weight-on-wheels transfer and affect normal reaction forces. The maximum braking efficiency for a dry level paved runway with tires and brakes in given condition (regulation dependent) for a fictitious FAR/CS 25 aircraft:

$$
\begin{aligned}
& \mu_{B}=\bar{\mu}_{b} \cdot \eta_{\text {BeffortMax }} \cdot \eta_{A B S} \cdot \eta_{\text {Weff }} \cdot \eta_{\text {TimeMax }} \approx \bar{\mu}_{b} \times 0.95 \times 0.90 \times 0.75 \times 0.95 \approx 0.61 \bar{\mu}_{b} \\
& \eta_{\text {BeffortMax }}<1 \quad \eta_{\text {ABS }}<1 \quad \eta_{\text {TimeMax }}<1 \quad \eta_{\text {Weff }}<1
\end{aligned}
$$

The antiskid efficiency factor $\eta_{A B S}$ could be distance (FAA, 2011) or timeaveraged ratio of the instantaneous to the peak braking force:

$\eta_{A B S}=\left[\int_{0}^{t} R_{B}(v) d t\right] /\left[\int_{0}^{t} R_{B, \max }(v) d t\right]$

According to FAR 25.109 (FAA, 2013), the maximum (peak) braking coefficient of friction is given in polynomial form for wet smooth paved runways with experimentally-derived maximum braking coefficients as a function of tire pressure. Similar polynomial form can be experimentally obtained for dry or contaminated runways and as a function of tire pressure and tire construction for given surface micro- and macro-texture. The general $5^{\text {th }}$-order polynomial form and the average maximum braking friction coefficient in the range of groundspeeds ( $V$ is in knots) from zero to arbitrary value is (FAA, 2011, 2013):

$\bar{\mu}_{b}=\frac{1}{v} \int_{0}^{v}\left[\sum_{n=1}^{6} c_{n}\left(\frac{v}{100}\right)^{n-1}\right] d v=\sum_{n=1}^{6} c_{n}(n-1) \frac{v^{n-1}}{100^{n}} \quad c_{n}=f\left(p_{\text {tire }}\right.$, wet $/$ dry $)$

These coefficients also depend on the runway texture, design, and construction. For example, if the average braking coefficient of friction (speed range 160 to 0 knots) on dry asphalt/concrete runway (given micro- and macrotexture) with 300-psi (20.42 bar) aviation tires is 0.50 , the maximum average friction braking coefficient $\mu_{B}$ is 0.305 . A 400,000 lbf airplane performing RTO, on dry level paved runway with no thrust reversers will generate average maximum braking force of about 121,838 lbf. The total deceleration/stopping factor $\mu_{S T P}$ depends also on the available reverse thrust, runway slope, and aerodynamic drag. While reverse thrust and slope are not accounted for in dry, level, and paved runway performance certification, ever-present aerodynamic drag is unavoidable. Simpler linear relationship between the peak braking and rolling coefficients with groundspeed is given in Daidzic and Shrestha (2008). 
The weight of the entire airplane cannot be on tires due to significant lift production even with spoilers deployed during RTO. The fully-modulated antiskid (anti-lock) systems are about 90\% efficient and that efficiency must be accounted for in AS test and certification runs (FAA, 2011). One also has to consider the entire braking dynamics and the possible braking torque limit (Durbin and Perkins, 1962; Mair and Birdsall, 1992). FAR 25 requires that brakes used in testing and certification runs be worn to the remaining $10 \%$ of their replacement limit. More in-depth modelling and simulation of deceleration runs as they apply to landing scenarios on dry, wet, and contaminated runways is given in Daidzic and Shrestha (2008). The average friction braking and the total deceleration force can be now written as $R_{B}=\mu_{B} \cdot W$ and $F_{S T P}=\mu_{S T P} \cdot W$.

If the runway is level, then the only additional forces to friction braking acting on an airplane are aerodynamic drug and thrust reversing. However, since the reverse thrust credit is not allowed during dry-runway certification, the idle forward residual thrust just about cancels, relatively small, deceleration caused by air resistance, and all what is left is friction braking. On wet and slippery runways, the friction (adhesion) braking can diminish significantly, often leaving thrust reversing as the major deceleration force (Daidzic and Shrestha, 2008). Many factors affect friction braking, including tire construction, tire dynamics, tire pressure, runway micro- and macro-texture, braking torque limit, crosswind, etc. Estimating friction braking forces is one of the most difficult problems in landing and RTO dynamics (Daidzic and Shrestha, 2008; Torenbeek, 2013).

\section{Accelerate-Stop distances}

The AS distance is calculated based on the Title 14 CFR 25.109 definitions and requirements (FAA, 2013) and T-category flight test guide AC 257C (FAA, 2012). This procedure is used for certification of field performance and only partially replicates real-life RTOs. FAA regulations stipulate no use of thrust reversers for dry runways. However, Amendment 25-92 (1998) allowed the use of thrust reversers during wet runway certification runs. Average acceleration and deceleration forces are assumed. Brakes used are worn out to within $10 \%$ of their usable limit. A sudden and complete failure with total loss of thrust on one engine is assumed at $v_{E F} \geq v_{M C G}$. Engine failure speed is related to takeoff decision/action speed by regulatory recognition time delay. Thus, for the short period $\Delta t_{1}$ as demonstrated or minimum per regulation (currently $1 \mathrm{~s}$ in FAR/CS 25) after the sudden engine failure, an airplane is accelerating (at reduced rate) under OEI conditions. At typical FAR/CS 25 airplane takeoff speeds this is about 200-300 $\mathrm{ft}(60-90 \mathrm{~m})$ and thus not entirely negligible. During the transition 
period, as illustrated in Figure 8, pilots will apply deceleration systems in a specific order (brakes-throttles-spoilers). This is the period during which the acceleration (and reaching the maximum transition speeds $V_{T R M A X}>V_{l}$ ) mutates into deceleration and is difficult to characterize. Distance covered during this transition period (acceleration and deceleration length) is equal to distance covered at constant $V_{l}$ speed at bit longer time:

$$
\Delta s_{\text {trans }}=\int_{t_{1}}^{t_{B}} v(t) d t=\bar{v} \cdot\left(t_{B}-t_{1}\right)=\int_{t_{1}}^{t_{2}} v_{1} d t=v_{1} \cdot\left(t_{2}-t_{1}\right)=v_{1} \cdot \Delta t_{2}
$$

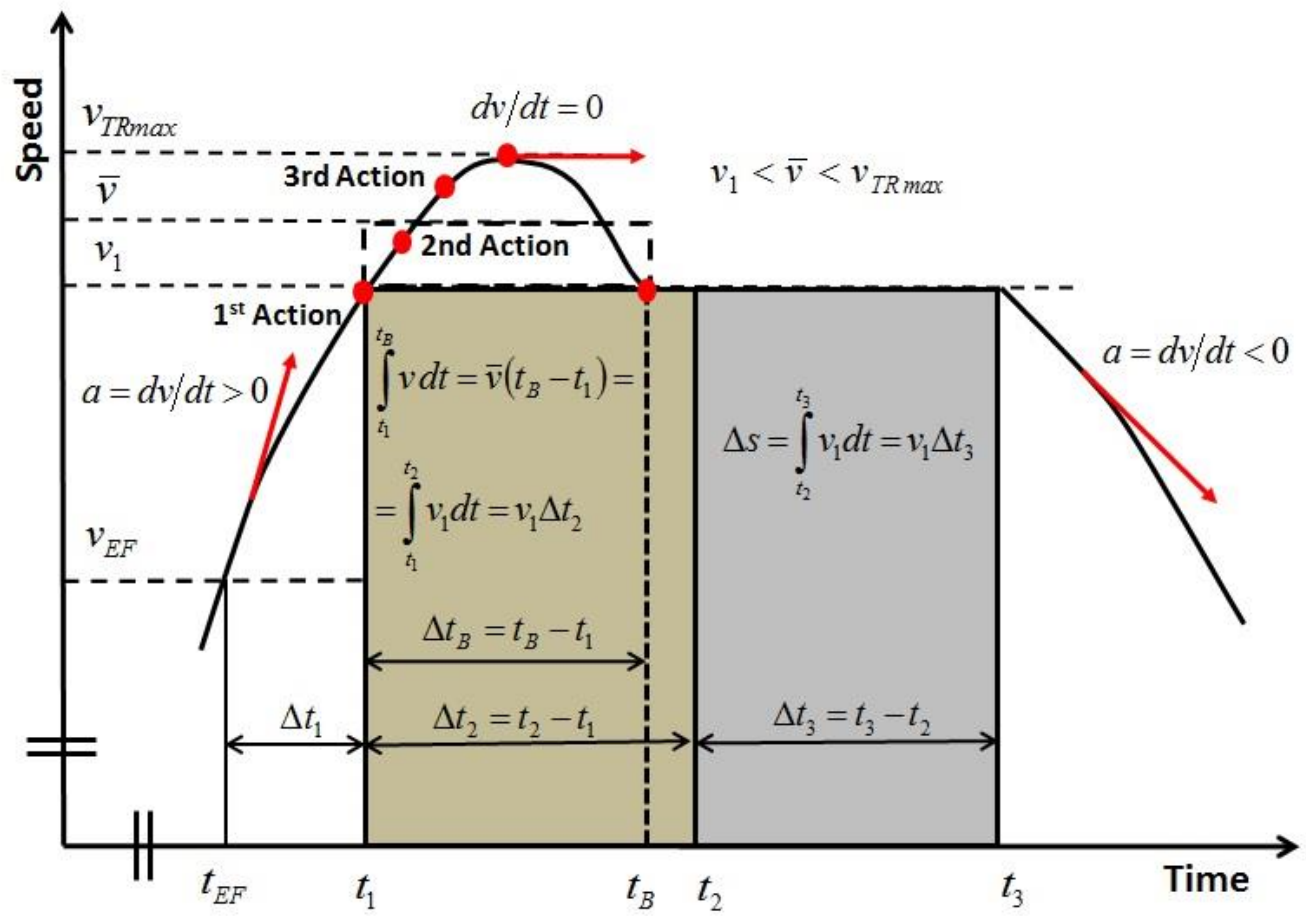

Figure 8. Illustration of the AS transition region with the $\Delta t_{1}=1 \mathrm{~s}$ engine-failure recognition time, flight testing demonstrated transition period $\Delta t_{B}$, and the mandatory $\Delta t_{3}=2 \mathrm{~s}$ coasting at $V_{l}$ as required by FAR 25.109. Not to scale.

The period $\left(t_{B}-t_{1}\right)$, during which sequential actions are taken by pilots to stop the aircraft, has to be demonstrated during flight testing. It is or 1 second or actual demonstrated time, whichever longer (FAR 25.109). The time $t_{B}$ designates the instant the speed drops back to $V_{l}$, after it reached the maximum transition speed $V_{\text {TRmax }}$. Since the acceleration/deceleration is constantly changing in 
transition period, an equivalent coasting period of $\Delta t_{2}>\Delta t_{B}=\left(t_{B}-t_{1}\right)$ at $V_{1}$ is used. Also, by regulations, the mandatory inertia period $\Delta t_{3}$ is added. Currently, it is two seconds (FAA, 2013) of coasting at takeoff decision/action speed $V_{l}$. Typically, such mandatory operational distances are in the range of $400-600 \mathrm{ft}$ (120-180 m) for FAR/CS 25 certified airplanes. Dynamics of takeoffs using total energy model was already discussed in many details in Daidzic (2014a) and Daidzic (2016b). The energy balance for AS-distance using EAS yields:

$\frac{v_{E F}^{2}}{2 \sigma a_{A E O}}+\frac{v_{1}^{2}-v_{E F}^{2}}{2 \sigma a_{O E I}}+\frac{v_{1}}{\sqrt{\sigma}} \Delta t_{2}+\frac{v_{1}}{\sqrt{\sigma}} \Delta t_{3}+\frac{v_{1}^{2}}{2 \sigma a_{S T P}}=A S D R \leq A S D A_{c o r r}$

where: $v_{1}=v_{E F}+\Delta v=v_{E F}+a_{O E I} \cdot \Delta t_{1}=v_{E F}+\frac{F_{O E I} \cdot g}{W} \Delta t_{1}$

We are using factored (net) distances required by regulations and thus gross (unfactored) ASD is always less than net (factored or scheduled) ASDR. The difference between ASD and ASDR comes from the inclusion of currently mandatory 2 seconds cruise-delay at $V_{l}$. This factoring reduces maximum weight and increases safety margins, $A S D R=A S D+\left(v_{1} / \sqrt{\sigma}\right) \Delta t_{3}$. The available distance for AS-maneuver is: $A S D A_{\text {corr }}=T O R A+S W Y-X_{B}-X_{R O L L}<A S D A$. Here, $X_{B}$ is the airplane make $\&$ model certified runway-alignment distance measured between the beginning of ASDA and the nose gear location (BRP). The rolling takeoff distance $X_{R O L L}$ is the equivalent distance that is being lost during rolling takeoff as compared to takeoff from standstill utilizing maximum takeoff thrust (MTT/TOGA). While $X_{R O L L}$ is zero for certification purposes, $X_{B}$ will be always finite and is conservatively about $200 \mathrm{ft}$ for wide-body and about $100 \mathrm{ft}$ for narrow-body FAR 25 T-category airplanes (Swatton, 2008). After lengthy reductions, we obtain the AS or ASDR energy equation in units of length:

$f_{1}\left(W, v_{1}\right)=\frac{1}{2 g A} \cdot W \cdot v_{1}^{2}+B \cdot v_{1}-\frac{C}{W}-E=0$

where,

$$
\begin{array}{cc}
A=\frac{F_{A E O} \cdot F_{S T P}}{F_{A E O}+F_{S T P}}>0 & B=\delta \cdot \Delta t_{1}+\sqrt{\sigma} \cdot\left(\Delta t_{2}+\Delta t_{3}\right)>0 \\
C=\frac{g\left(\Delta t_{1}\right)^{2}}{2} \delta \cdot F_{O E I}>0 & E=\sigma \cdot A S D A_{\text {corr }}>0
\end{array}
$$


with, $F_{S T P}=\mu_{S T P} \cdot W, \Delta t_{1}=1 \mathrm{~s}, \Delta t_{2}=2 \mathrm{~s}, \Delta t_{3}=2 \mathrm{~s}$, and

$$
\delta=1-\left(\frac{a_{O E I}}{a_{A E O}}\right)=1-\left(\frac{F_{O E I}}{F_{A E O}}\right)=\frac{F_{A E O}-F_{O E I}}{F_{A E O}}<1
$$

AS transition time $\Delta t_{2}$ was arbitrarily chosen to be two seconds to simulate flight test data. Three kind of net average forces are affecting airplane during AS maneuver: AEO net accelerating force, slowing down or braking deceleration (stopping) force, and, to a very short duration, OEI net accelerating force. The average acceleration coefficient $A$ is weight-dependent and weight iterations combined with the nonlinear solver are required. To simulate the case of AS due to an event (e.g., tire failure) or AEO AS RTO, one only needs to set $\Delta t_{1}=0$, which leads to $C=0$ and diminished $B$. In that case, deceleration may be seriously affected. Even simpler equation for AS maneuver is obtained if the transition and coasting periods at $V_{l}$ are neglected, i.e., $B=0$. While this will definitely not be very accurate in determining $V_{l}$ and maximum $W$ from available AS-distance it gives good physical insight into no-wind RTO physics:

$$
v_{1, A S}=\frac{\sqrt{\sigma} \cdot \sqrt{2 g \cdot A S D A_{c o r r}}}{\sqrt{W}}\left(\frac{F_{A E O} \cdot F_{S T P}}{F_{A E O}+F_{S T P}}\right)^{1 / 2} \quad v_{1, A S} \propto \frac{1}{\sqrt{W}}
$$

Both, high acceleration and high deceleration, forces enable higher stopping-onset speeds. On contaminated runways, the AEO net acceleration remains mostly unchanged, while the stopping force are significantly diminished, resulting in lower $V_{l}$. However, occasionally extra drag (impingement drag, slush, etc.) during accelerating ground roll lowers the net AEO accelerating force. On high-quality paved dry runways both, accelerating and decelerating, forces are high, resulting in high $V_{l}$ for given distance and weight. It also follows that $\left(\partial v_{1} / \partial W\right)_{A S D A}<0$, and $\left[\partial v_{1} / \partial(A S D A)\right]_{W}>0$. The essential functional relationships are illustrated in Figure 9 (AS=ASDA, TO=TODA/TORA). To calculate Jacobian and use Newton-Raphson solvers (Appendix B), the first-order partial derivatives of $f_{1}$ are evaluated, i.e., $\left(\partial f_{1} / \partial W\right)_{v_{1}}$, and $\left(\partial f_{1} / \partial v_{1}\right)_{W}$. After formally evaluating $2^{\text {nd }}$-order partial derivatives (including cross-derivatives) of $f_{1}$, i.e., $\left(\partial^{2} f_{1} / \partial x_{i} \partial x_{j}\right)$, we found that Hessian $|H|<0$ (Appendix C). It was determined that the first-order partial derivatives have no zeros in the positive real range of speeds and weights, thus no maximums exist. That also makes sense since in unconstrained optimization the airplane's kinetic energy can be made as high as possible by increasing TOW and $V_{l}$ without limit. 


\section{OEI accelerate-go takeoff distance}

The energy-balance for continuing (AG) takeoff (FAR/CS 25.113) after sudden engine failure just past $V_{E F}$ and decision made to continue at $V_{l}$, yields:

$\frac{v_{E F}^{2}}{2 \sigma a_{A E O}}+\frac{v_{L O F}^{2}-v_{E F}^{2}}{2 \sigma a_{O E I}}+\left(\frac{W}{T_{O E I}-D_{a}}\right)\left(\frac{v_{S H}^{2}-v_{L O F}^{2}}{2 \sigma g}+S H\right)=T O D R \leq T O D A_{c o r r}$

where, $v_{E F}=v_{1}-a_{O E I} \cdot \Delta t_{1}$, and, TODA $A_{\text {corr }}=T O R A+C W Y-X_{A}-X_{R O L L}<T O D A$

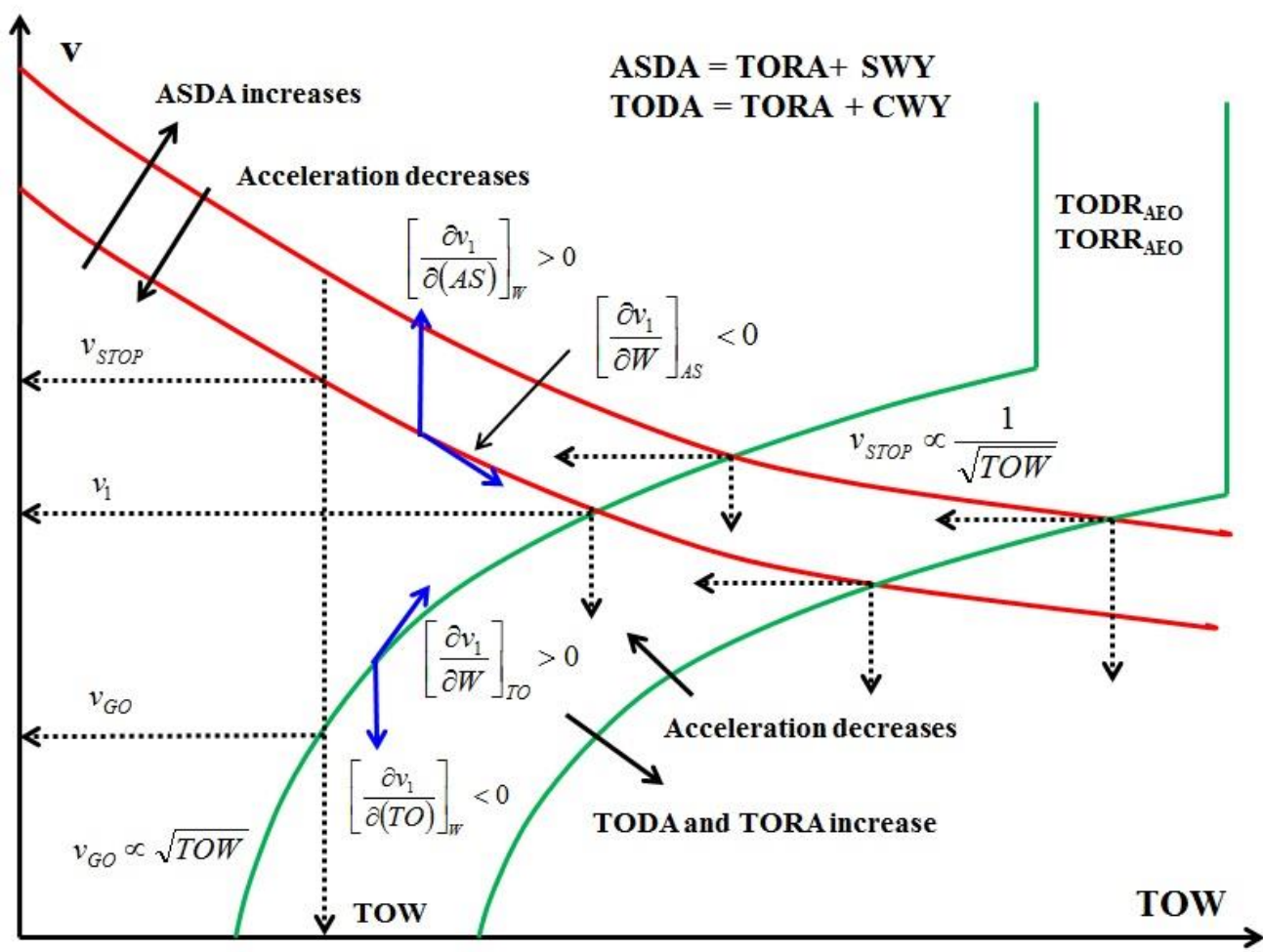

Figure 9. The essentials of T-category airplane's takeoff physics. Not to scale.

The gross TOR is equal to net TORR, as no safety factorization is taken for one-in-a-million OEI AG maneuver. The average OEI angle of climb (climb gradient) during airborne takeoff part is:

$\frac{1}{\bar{\gamma}_{T O, O E I}}=\left(\frac{W}{T_{O E I}-D_{a}}\right)_{\text {avg,TO }}$ 
By using extremal value and performing tedious mathematical reductions, we obtain the AG TODR energy equation in units of length:

$f_{2}\left(W, v_{1}\right)=\frac{1}{2 g G_{1}} \cdot W \cdot v_{1}^{2}-H_{1} \cdot v_{1}+\frac{K_{1}}{W}-P_{1} \cdot W^{2}-R_{1} \cdot W+U_{1}=0$

where:

$G_{1}=\frac{F_{A E O} \cdot F_{O E I}}{F_{A E O}-F_{O E I}}=\frac{F_{O E I}}{\delta}>0 \quad H_{1}=\frac{F_{O E I} \cdot \Delta t_{1}}{G}>0$

$K_{1}=\frac{F_{O E I}^{2} \cdot g \cdot\left(\Delta t_{1}\right)^{2}}{2 \cdot G}>0 \quad L_{1}=g \cdot \rho_{S L} \cdot S_{r e f} \cdot C_{L, T O \max }>0$

$M_{1}=\frac{\xi_{L O F}^{2}}{L_{1} \cdot F_{O E I}} \quad N_{1}=\frac{\xi_{S H}^{2}-\xi_{L O F}^{2}}{L_{1} \cdot\left(T_{O E I}-D_{a}\right)} \quad P_{1}=M_{1}+N_{1}$

$R_{1}=\frac{\sigma \cdot S H}{\left(T_{\text {OEI }}-D_{a}\right)}>0 \quad U_{1}=\sigma \cdot T O D A_{c o r r}>0$

The main-gear runway line-up correction, $X_{A}$ is conservatively about 100 $\mathrm{ft}$ for wide-body and about $50 \mathrm{ft}$ for narrow-body T-category airplanes (Swatton, 2008). Runway alignment corrections are certified for every airplane type (Swatton, 2008). The liftoff and screen-height airspeeds (EAS) are expressed as:

$v_{L O F}=\xi_{L O F} \sqrt{\frac{2 W}{\rho_{S L} S_{r e f} C_{L, T O \max }}} \quad v_{S H}=\xi_{S H} \sqrt{\frac{2 W}{\rho_{S L} S_{r e f} C_{L, T O \max }}}$

The product of the constant geometric wing-reference surface $S_{\text {ref }}$ and the maximum coefficient-of-lift in takeoff configuration $C_{L, T O m a x}$, defines $S_{\text {eff }}$ or the effective lifting surface; the larger the effective lifting surface the lower the stalling speed. There is a direct relationship between the steady-state coefficientof-lift at LOF and the maximum (stalling) coefficient-of-lift in the takeoff configuration, $C_{L, T O, L O F}=C_{L, T O \max } / \xi_{L O F}^{2}$. There is a bit of uncertainty with the factors for $V_{L O F}$ and $V_{S H}$ speeds and due to various stability and control issues during rotation and transition to flight, the manufacturer can use different operational values of these speeds. However, all these aforementioned speeds have to meet various restriction as defined by FAR/CS 25.107. 
A minimum takeoff safety speed $V_{2 M I N}$ cannot be less than $1.2 V_{S I}$ or 1.13 $V_{S R I}$. We used this criteria to define OEI SH-speed which is identical to the takeoff safety speed, i.e., $V_{S H}=V_{2}$ speed. Liftoff speed occurs between the rotation $V_{R}$ and the OEI SH-speed $V_{S H}$, and must be always faster than flight-test determined $V_{M U}$ (minimum-unstick airspeed). Deceleration forces play no role in AG maneuver. A special case exists if the short engine-failure recognition delay is neglected. In that case the takeoff decision/action speed can be expressed analytically as (see also Figure 9):

$v_{1, A G} \approx \sqrt{2 g G_{1} R_{1}} \cdot \sqrt{1+\left(\frac{P_{1}}{R_{1}}\right) \cdot W-\left(\frac{U_{1}}{R_{1}}\right) \cdot \frac{1}{W}} \propto \sqrt{W}$

The $1^{\text {st }}$ - and $2^{\text {nd }}$-order partial derivatives of $f_{2}$ are formally derived. As expected, it was found that $V_{l} \mathrm{~s}$ and TOWs have no extremums in the positive real range. The coefficient $K_{1}$ is on the order of $10^{5}$ and $P_{1}$ is on the order of $10^{-7}$. However, weight is also on the order of $10^{5}$ and $\mathrm{W}^{3}$ is thus on the order of $10^{15}$. The $2^{\text {nd }}$ derivative with respect to weight is thus negative and of opposite sign of $2^{\text {nd }}$ derivative over airspeed. The Hessian (Appendix C) is again negative. From basic considerations, we now have, $\left(\partial v_{1} / \partial W\right)_{T O D A}>0$, and $\left[\partial v_{1} / \partial(T O D A)\right]_{W}<0$. Using the Newton-Raphson (N-R) solver for the system of nonlinear equations, the real roots for $V_{l}$ and $W$ can be calculated. Our problem of finding $V_{l}$ and the maximum $W$ (TOW) for the given conditions is relatively good behaved with overall rapid convergence. For more information on various $\mathrm{N}-\mathrm{R}$ and other nonlinear solvers for equations with constant coefficients (algebraic and transcendental) consult Press et al. (1992) and Demidovich and Maron (1987).

\section{One-engine-inoperative takeoff run weight net limit}

As CWY length increases, OEI TORR may limit FLLTOW. The energy balance for the TORR required under the FAR 25.113(c)(1)(i) for dry runway (SH of $35 \mathrm{ft}$ ), yields:

$$
\frac{v_{E F}^{2}}{2 \sigma a_{A E O}}+\frac{v_{L O F}^{2}-v_{E F}^{2}}{2 \sigma a_{O E I}}+\frac{1}{2}\left(\frac{W}{T_{O E I}-D_{a}}\right)\left(\frac{v_{S H}^{2}-v_{L O F}^{2}}{2 \sigma g}+S H\right)=T O R R
$$

where: $T O R=T O R R \leq T O R A_{c o r r}$, and, TORA $A_{c o r r}=T O R A-X_{A}-X_{R O L L}<T O R A$.

Similarly, as in the TODR case, we obtain AG TORR energy equation: 
$f_{3}\left(W, v_{1}\right)=\frac{1}{2 g G_{2}} \cdot W \cdot v_{1}^{2}-H_{2} \cdot v_{1}+\frac{K_{2}}{W}-P_{2} \cdot W^{2}-R_{2} \cdot W+U_{2}=0$

where,

$G_{2}=\frac{F_{A E O} \cdot F_{O E I}}{F_{A E O}-F_{O E I}}=\frac{F_{O E I}}{\delta}>0 \quad H_{2}=\frac{F_{O E I} \cdot \Delta t_{1}}{G_{2}}>0$

$K_{2}=\frac{F_{O E I}^{2} \cdot g \cdot\left(\Delta t_{1}\right)^{2}}{2 \cdot G_{2}}>0 \quad L_{2}=g \cdot \rho_{S L} \cdot S_{r e f} \cdot C_{L, T O \max }>0$

$M_{2}=\frac{\xi_{L O F}^{2}}{L_{2} \cdot F_{O E I}} \quad N_{2}=\frac{\xi_{S H}^{2}-\xi_{L O F}^{2}}{2 \cdot L_{2} \cdot\left(T_{O E I}-D_{a}\right)} \quad P_{2}=M_{2}+N_{2}$

$R_{2}=\frac{\sigma \cdot S H}{2 \cdot\left(T_{\text {OEI }}-D_{a}\right)}>0 \quad U_{2}=\sigma \cdot$ TORA $A_{\text {corr }}>0$

If TORR is more limiting than TODR, the nonlinear system of equations defining ASDA and TORA field-length limits is solved using the same N-R solver, which results in the maximum TOW and corresponding $V_{l}$.

\section{All-engine takeoff distance and takeoff run weight net limits}

Takeoff can be also limited by the net (factored) AEO scenario. In that case a safety factor of $15 \%$ is currently added to demonstrated gross distance. The $15 \%$ add-on comes from the study of operating variations (Eshelby, 2000) resulting in 3\% standard deviation (SD) and five SDs are taken to account for slightly less than one-in-a-million $\left(10^{-6}\right)$ probability of not meeting the operational requirements. Thus, for the AEO TODR case, we have:

$1.15 \cdot\left[\frac{v_{L O F}^{2}}{2 \sigma a_{A E O}}+\left(\frac{W}{T_{A E O}-D_{a}}\right) \cdot\left(\frac{v_{3}^{2}-v_{L O F}^{2}}{2 \sigma g}+S H\right)\right]=T O D R_{A E O} \leq T O D A_{c o r r}$

After mathematical reductions, we obtain the quadratic equation for AEO factored field-length limited unknown maximum takeoff weight:

$a_{1} \cdot W^{2}+b_{1} \cdot W+c_{1}=0$

where, 


$$
a_{1}=\frac{\xi_{L O F}^{2}\left[\frac{1}{F_{A E O}}+\frac{\left(\xi_{3} / \xi_{L O F}\right)^{2}-1}{\left(T_{A E O}-D_{a}\right)}\right]}{g \rho_{S L} S_{r e f} C_{L, T O \max }} \quad b_{1}=\frac{\sigma \cdot S H}{\left(T_{A E O}-D_{a}\right)} \quad c_{1}=-\frac{\sigma \cdot T O D A_{c o r r}}{1.15}
$$

The net AEO takeoff weight limit is the smaller positive real value. Naturally, no complex-conjugate or negative real solutions are allowed. The airspeed during AEO takeoff and at SH (35 ft dry or $15 \mathrm{ft}$ wet) is not defined. In operating practice $V_{3}$ is typically $V_{2}$ plus $10-20$ knots. We could introduce another energy equation to estimate $V_{3}$ more accurately, but that would add to complexity and we simply assume that speed at SH during AEO takeoff is $V_{3}=1.3 V_{S 1}$. Twinengine jets are normally field-length limited by OEI (gross performance) takeoffs. Only very lightly loaded T-category twin-jets may become net-AEO field limited. Conversely, four-engine jets are often field-limited by the $115 \%$ AEO takeoff (net) factorization. Tri-jets are often restricted by $V_{R}$ limit (Swatton, 2008).

As CWY becomes longer, the takeoff run FLLTOW becomes more important and at some critical CWY length it is limiting takeoff mass/weight. For factored AEO takeoff scenario according to FAR/CS 25.113(c)(1)(ii) for dry runway case we have the mathematical condition:

$$
1.15 \cdot\left[\frac{v_{L O F}^{2}}{2 \sigma a_{A E O}}+\frac{1}{2}\left(\frac{W}{T_{A E O}-D_{a}}\right) \cdot\left(\frac{v_{3}^{2}-v_{L O F}^{2}}{2 \sigma g}+S H\right)\right]=T O R R_{A E O} \leq T O R A_{c o r r}
$$

After rearrangements, we obtain similar condition to AEO TODA:

$$
a_{2} \cdot W^{2}+b_{2} \cdot W+c_{2}=0
$$

where,

$$
a_{2}=\frac{\xi_{L O F}^{2}\left[\frac{1}{F_{A E O}}+\frac{\left(\xi_{3} / \xi_{L O F}\right)^{2}-1}{2\left(T_{A E O}-D_{a}\right)}\right]}{g \rho_{S L} S_{r e f} C_{L, T O \max }} \quad b_{2}=\frac{\sigma \cdot S H}{2\left(T_{A E O}-D_{a}\right)} \quad c_{2}=-\frac{\sigma \cdot T O R A_{c o r r}}{1.15}
$$

It is to be expected that in the case of absence or very short CWYs, the FLLTOW will be limited by TODA. In the case of sufficiently long CWY, the FLLTOW will become TORA limited. One could now explore many different dry, wet, or contaminated runway takeoff scenarios by changing regulatory required $\mathrm{SHs}$ (e.g., 0, 15, or $35 \mathrm{ft}$ ) and $\mathrm{SH}$ speeds $\left(V_{S H}\right)$. The effect of changing 
TORA and TODA on $V_{l}$ are illustrated in Figure 9. Changing $\mathrm{SH}$ will affect required TOD/TOR airborne part and accordingly TODA/TORA. This will be especially interesting when evaluating wet and contaminated runway takeoffs which is planned in a future contribution.

\section{Calculation of $V_{1}$-range when TOW is less than FLLTOW}

In the case that actual TOW is less than FLLTOW three options are possible (Daidzic, 2012b, 2013b, 2014a):

1. Reduced/derate (flexible) thrust takeoff is conducted, while possibly rebalancing the runway.

2. Range of $V_{l}$ speeds exist between $V_{S T P}$ and $V_{G O}$.

3. Overspeed takeoffs (with or without reduced/derated thrust) are conducted to increase climb-limited weights, while possibly rebalancing the runway.

We are now interested in determining the range of $V_{l}$ speeds when TOW is less than FLLTOW. In fact, theoretically, TOW can also be larger than FLLTOW, but that results in unsafe takeoff condition. The maximum possible speeds that will still satisfy ASDA limitation are designated as $V_{S T P}$ speeds and can be estimated from:

$\left(\frac{W}{2 g A}\right) \cdot v_{S T P}^{2}+B \cdot v_{S T P}-\left(\frac{C}{W}+E\right)=0$

The minimum takeoff speeds $V_{G O}$ from which the airplane can still perform OEI AG and meet TODA restriction are evaluated from:

$\left(\frac{W}{2 g G_{1}}\right) \cdot v_{G O}^{2}-H_{1} \cdot v_{G O}+\left(\frac{K_{1}}{W}-P_{1} \cdot W^{2}-R_{1} \cdot W+U_{1}\right)=0$

Similar quadratic expression exists when TORA is more limiting. As the TOW approaches FLLTOW, $V_{S T P}$ decreases to and $V_{G O}$ increases toward unique $V_{l}$. Therefore, at all times it must be, $v_{M C G}<v_{G O} \leq v_{1} \leq v_{S T P} \leq v_{M B E}$. It is very likely that at light weights, $v_{R} \leq v_{S T P}=v_{1, \max }$. However, if overspeed takeoffs are conducted, higher rotation speeds can be chosen (Daidzic, 2014a). A tire speed may interfere with the improved- $V_{2}$ takeoffs, such that, $v_{L O F} \leq v_{\text {TIRE }}$. The range of $V_{l}$ speeds is illustrated in Figure 9. If TOW > FLLTOW, a range of speeds exists for which an airplane cannot stop or go, should the engine fail there. 


\section{Additional constraints for takeoff computations and optimization}

There are some very important stability, control, and performance limitations that are constraining the choice of $V_{l}$ and FLLTOW during takeoffs. The OEI asymmetric thrust during ground roll must be offset by rudder and nose gear tire cornering side force resulting in $v_{1}-a_{O E I} \cdot \Delta t_{1}=v_{E F} \geq v_{M C G}$.

The maximum attainable $V_{1}$ must be less than the maximum braking energy (MBE) that can be dissipated by aircraft brakes and is expressed in terms of groundspeed, $v_{1} \leq v_{M B E}$. Additionally, in the case of continuing OEI takeoff the maximum tire groundspeed should not be exceeded, i.e., $v_{L O F} \leq v_{\text {TIRE }}$. The structural takeoff weight limitation implies $F L L T O W \leq M S T O W$. Although it seems rather obvious, the following constraints must be satisfied as well, $v_{1} \geq 0$ and $W \geq 0$. A twin-engine airplane in takeoff configuration that lifts off and retracts landing gear should be able to achieve OEI still-air $2.4 \%$ climb gradient at $V_{2}$ using 5- or 10-minutes maximum takeoff power (Padilla, 1996). This climblimited TOW (CLTOW), formerly known as WAT limit (Weight-AltitudeTemperature), could lower RTOW when airplane is out of ground effect due to high DA:

$F L L T O W \leq C L T O W \approx \frac{T_{O E I}(\sigma)-D\left(W, C_{D}\right)}{0.024}$

The case of improved or overspeed- $V_{2}$ takeoffs, where flying faster decreases vortex-drag, has been discussed in Daidzic (2014a). The case of obstacle-limited TOW (OLTOW) and cruise and landing TOW limits are not included, as they are route and departure/destination airport specific.

\section{The effect of air density and flap setting on takeoff distances}

Air density plays crucial role in takeoffs. Two important adverse effects exists. First, the TAS increases for the same EAS as air density is decreased (increased elevation, temperature, humidity, and/or lower barometric air pressure or all effects combined). Second, jet engines are air-breathing propulsion devices and in the first approximation it can be assumed that thrust decreases linearly with air density. The takeoff distance assuming constant acceleration up to $V_{2}$ is:

$s_{G}=\frac{(1.2)^{2}}{\rho_{S L} g} \cdot \frac{\left(W / S_{r e f}\right)}{\sigma^{2} C_{L, T O \max }(F / W)_{S L, I S A}} \propto \frac{W^{2}}{\sigma^{2} S_{e f f} F_{S L, I S A}}$ 
with, $S_{\text {eff }}=S_{r e f} \cdot C_{L, T O \max } \quad F_{S L, I S A} \approx T_{S L, I S A}-(D+R+\phi W)$

Hale (1984) uses gross SL thrust-to-weight (T/W) ratio in Equation (27) which underestimates the required distances as it does not account for retarding forces. Theoretically, at $7,400 \mathrm{ft}$ ISA where $\sigma$ is approximately 0.8 , the ground run alone will be about $56 \%$ longer. There are other propulsive effects (TAS effect and increase in momentum drag) that will cause takeoff distance to increase even more due to lower air density (Daidzic, 2012b, 2016c). DA calculations and resulting graphs are presented in Appendix D.

A statistical method to estimate AEO takeoff distance of FAR/CS 25 Tcategory yields (Loftin, 1980; Roskam and Lan, 1997):

$$
S_{\text {TODR }}=37.5 \times \text { TOP }_{25} \quad \text { TOP }_{25}=\frac{(W / S)_{T O}}{\sigma^{2} C_{L, T O \max }(T / W)_{T O, S L}}
$$

Straight application of Equation (27) would result in constant multiplier of 21.65 as $115 \%$ of gross distance is used for AEO TODR. Consider that Equation (27) uses net T/W ratio, while Equation (28) utilizes rated gross SL T/W. If we assume that net takeoff thrust (gross thrust minus all resistances) is about $75 \%$ of the gross takeoff thrust, we obtain multiplier of about 29, which is still $23 \%$ lower than what semi-empirical Equation (28) predicts. Loftin (1980) shows that many production commercial multi-engine airplanes follow the linear correlation line. Accordingly and using Equation (28), if our fictitious T-category airplane has wing-loading (W/S) of $132 \mathrm{lbf} / \mathrm{ft}^{2}$, maximum TO lift-coefficient of 2.2, net SL takeoff T/W ratio of 0.3 , and taking off at the DA of $3,600 \mathrm{ft}$, the $\mathrm{TOP}_{25}$ parameter becomes $247 \mathrm{ft}$ and the FAR takeoff distance is accordingly 9,260 ft.

Increased flap setting increases $C_{L}$ (effective lifting surface) lowering stalling and other performance/control airspeeds, but it also increases coefficient of drag $C_{D}$ (both parasitic and induced) possibly seriously impairing airborne takeoff climb performance. And while the airplane may become airborne earlier and climb shallower in ground effect to $\mathrm{SH}$, once it leaves the ground effect it may not be able to meet required climb performance. The effect of flap setting on takeoff dynamics is discussed in Daidzic (2014a).

\section{Sensitivity due to small changes in weight, runway length, and air density}

A question arises of how much small uncertainties in actual takeoff weight, runway length, and air density affect takeoffs. Since we are only talking 
about the small linear perturbations around equilibrium points, Taylor's first-order expansion of multivariable functions (e.g., Appendix C) will be used. The sensitivity (or uncertainty) of liftoff-speed on aircraft weight and air density (air pressure and temperature) can be expressed as:

$$
\frac{\Delta v_{L O F}}{v_{L O F}}=\frac{1}{2} \frac{\Delta W}{W}-\frac{1}{2} \frac{\Delta \sigma}{\sigma}=\frac{1}{2} \frac{\Delta W}{W}-\frac{1}{2} \frac{\Delta \delta}{\delta}+\frac{1}{2} \frac{\Delta \theta}{\theta}
$$

Thus, a $10 \%$ increase in TOW will result in 5\% increase in liftoff speed. A $5 \%$ decrease in air pressure together with $5 \%$ increase in air temperature will cause $10 \%$ decrease in air density. A $10 \%$ decrease in air density will cause $5 \%$ increase in liftoff TAS (EAS stays the same). From Equation (29) we can estimate the inverse effect of liftoff speed and air pressure and temperature on TOW.

\section{Runway distance correction due to wind}

The effect of wind was not included in the mathematical model. The effect of crosswind (XW) on tire dynamics is neglected as well. Only the wind component co-linear with the runway is used. Wind not only changes GS of the aircraft, but also the time exposure to it. Headwind (HW) is by definition positive (HW > 0), while tailwind (TW) is negative (TW < 0). Distance covered in ground roll under steady wind along the runway using aircraft's TAS is (Durbin and Perkins, 1962; Mair and Birdsall, 1992; Saarlas, 2007; Vinh, 1993):

$$
s_{G w}=\int_{v_{w}}^{v_{L O F}} \frac{v d v}{a}-v_{w} \int_{v_{w}}^{v_{L O F}} \frac{d v}{a}=\frac{\left(v_{L O F}-v_{w}\right)^{2}}{2 \cdot \bar{a}}
$$

The speed- and time-averaged accelerations in above integrals are not the same as for no-wind situation (Mair and Birdsall, 1992). The average acceleration is taken from the point where TAS is equal to steady wind component (HW or TW) to the liftoff TAS. Since acceleration is not constant during takeoff, the average acceleration will be slightly different in the no-wind, HW, or TW cases. However, for small wind factors we can neglect those differences. The effect of wind on ground roll distance with airplane's CAS/EAS liftoff airspeed in the first approximation for small wind factors is proportional to:

$$
s_{G w} \propto\left(\frac{v_{L O F}}{\sqrt{\sigma}}-v_{w}\right)^{2}=\frac{v_{L O F}^{2}}{\sigma} \cdot\left\{\left[1+\sigma \cdot\left(\frac{v_{w}}{v_{L O F}}\right)^{2}\right]-2 \cdot\left(\frac{v_{w}}{v_{L O F}}\right) \cdot \sqrt{\sigma}\right\}=s_{G 0} \cdot F_{w}
$$


We assumed that wind is reported in terms of true airspeed and true or magnetic direction as appropriate in different situations. Dynamic pressure created by wind will depend on the existing air density. The no-wind distance is always increased by dimensionless wind factor (normally quite small) as the $2^{\text {nd }}$ term above illustrates. The $3^{\text {rd }}$ term in the square parenthesis above will be negative with HW and positive for TW. Consider also that regulations FAR $25.105(\mathrm{~d})(1)$ (FAA, 2013) require not more than $50 \%$ of reported $\mathrm{HW}$ to be included in computations and not less than $150 \%$ of the reported TW, resulting in two scenarios with factored reported winds:

$$
\begin{aligned}
& H W: \quad s_{G w} \propto s_{G 0} \cdot\left\{\left[1+\frac{\sigma}{4} \cdot\left(\frac{v_{w}}{v_{L O F}}\right)^{2}\right]-\left(\frac{v_{w}}{v_{L O F}}\right) \cdot \sqrt{\sigma}\right\} \\
& T W: \quad s_{G w} \propto s_{G 0} \cdot\left\{\left[1+2.25 \cdot \sigma \cdot\left(\frac{v_{w}}{v_{L O F}}\right)^{2}\right]-3 \cdot\left(\frac{v_{w}}{v_{L O F}}\right) \cdot \sqrt{\sigma}\right\}
\end{aligned}
$$

For example, a wind ratio of 0.1 (e.g., 16 knot along runway wind at $V_{L O F}$ $=160$ knots) for SL density will result in a ground-roll correction multipliers of 0.9025 and 1.3225 for HW and TW respectively. For HW, the ground roll is reduced, while for TW it is significantly increased. A 7,000 ft no-wind distance to liftoff will increase by about $32.3 \%$ due to 16 knot TW. The factored distance is now amazing 9,258 ft. Such wind factoring introduces additional inherent safety margins built into performance charts and it is illegal for an operator to take advantage of it (Swatton, 2008). During the airborne takeoff portion the airplane will accelerate to $V_{2}$, but the wind will slightly change due to Earth's boundary layer. More details on takeoff airborne phase wind accounting and wind gradient can be found in Durbin and Perkins (1962). More on the many curious effects of wind affecting aircraft in flight can be found in Daidzic (2016a). Mair and Birdsall (1992) and Vinh (1993) give particularly good in-depth discussion of wind effects on airplane's field performance. Also, McCormick (1995) gives good discussion and solution methods for inclusion of wind effects.

\section{Runway distance correction due to effective runway gradient}

Most commercial use runways have effective gradient (grade, slope) less than $\pm 2 \%$. Regulations, such as FAR 25.105(d)(2), require considerations of effective runway gradients for field performance calculations. The effective runway gradient is also defined in AC 150/5325-4B (FAA, 2005). We use linear perturbations to extract the runway slope correction on calculated TODR, ASDR, 
and TORR. The runway slope affects the longitudinal forces and accelerations. It can be treated in terms of the gravity-assisted thrust (downslope takeoff, $\Delta \phi<0$ ) or gravity-generated drag (upslope takeoff, $\Delta \phi>0$ ). During RTO's those functions are reversed and upslope runway is very helpful. Thus, we can write for the runway gradient effect alone:

$$
\frac{\Delta s}{s_{0}}=-\frac{\Delta a}{a_{0}} \quad \Delta a=\left(\frac{\partial a}{\partial \phi}\right)_{0} \Delta \phi=-g \cdot \Delta \phi
$$

Hence, the effective runway gradient (slope) correction is:

$$
F_{\phi}=\frac{s_{0}+\Delta s}{s_{0}}=1+\left[\frac{2 \cdot s_{0}(\sigma) \cdot g}{v_{1}^{2} / \sigma}\right] \cdot \Delta \phi
$$

For example, if an airplane lifts-off at 160 knots at SL density (EAS=CAS=TAS) and needs 7,000 ft of dry, level, and paved runway, what is the distance correction for average $2 \%$ upslope grade? After substituting values with $g=32.174 \mathrm{ft} / \mathrm{s}^{2}\left(g=9.80665 \mathrm{~m} / \mathrm{s}^{2}\right)$, one obtains the runway gradient correction of 1.1234 or about $12.3 \%$ longer liftoff run (now 7,864 ft) is required than for level runway under zero-wind and given SL conditions. The inclusion of density ratio in Equation (34) may be confusing. Increased TAS at higher DA to generate required EAS hints to decreasing effective runway gradient effect. However, consider that with decreasing density ratio, the runway required increases, and this is already factored in our main results. So the runway slope correction must be applied for the appropriate density ratio (DA). It is recommended that first the correction for wind is made and then the correction for effective runway slope (Durbin and Perkins, 1962). From the earlier wind-correction example, the 16knot TW correction increased the runway by about $32 \%$. This increased value should then be used when making runway gradient correction. Taking the example above and adding $12.34 \%$ to $9,257.5 \mathrm{ft}$, results in almost exactly 10,400 $\mathrm{ft}-\mathrm{a} 3,400 \mathrm{ft}$ increase of liftoff distance due to relatively gentle $+2 \%$ upslope and quite significant 16 knot TW. The combination of even mild TW, upslope runway, and high DA is absolutely devastating for an airplane conducting OEI AG takeoff.

\section{Results and Discussion}

The total-energy based mathematical model and TPT enable quite accurate estimation of FLLTOW and all takeoff airspeeds for balanced (BFC) and 
unbalanced takeoff (UBFC) conditions. Takeoff computations will depend on many factors, such as, air density (elevation, air pressure, temperature, and humidity), wind (direction and intensity), effective runway slope and surface quality, tire quality and pressure, dry, wet or contaminated runways, thrust setting, AEO and OEI cases, and a plethora of other more or less influential factors. To explore relative effects of each of these factors in a single article would be overwhelming.

The airplane used for simulations is a fictitious wide-body T-category airplane similar to Boeing's B767-300ER (Boeing, 2005). The MTOW is 410,000 lbf $(186,364 \mathrm{~kg})$, wing reference area of $3,100 \mathrm{ft}^{2}\left(288 \mathrm{~m}^{2}\right)$, wing span of $160 \mathrm{ft}$ $(\mathrm{AR}=8.26)$, and two flat-rated high-bypass turbofans with SL ISA 61,500 lbf $(273.55 \mathrm{kN})$ thrust. Such turbofan is in the same category with GE CF6-80C2B64, PW4060, and RR RB 211-524H engines. More details on this fictitious airplane together with drag polars for different configurations are given in Daidzic (2016c). Comparison with Boeing's published basic performance (D6-58328) data for airport planning (Boeing, 2005) was used to validate our physics-based takeoff performance model. Also, medium-range airplane data (CAA, 2006) resembling narrow-body medium-range $\mathrm{T}$-category $\mathrm{B} 737-400$ was used to validate TPT calculations.

The TPT is implemented in Fortran 90/95/2003/2008, Matlab, True Basic, and MS Excel software platforms. The accuracy of numerical computations exceeds 14 significant digits which in respect of many inherent uncertainties is not really necessary. To illustrate the process and how N-R solver is implemented let us choose a particular UBFL case shown in Figure 10.

The basic TORA is $8,000 \mathrm{ft}$ with $300-\mathrm{ft}$ SWY and $800-\mathrm{ft}$ CWY. The mainand nose-gear runway alignment (line-up) corrections are $100\left(X_{A}\right)$ and $200\left(X_{B}\right) \mathrm{ft}$ respectively. This is the case of no-wind, dry, hard-surfaced runway with required $\mathrm{SH}$ of $35 \mathrm{ft}$ at environmental relative density of 0.9 (about 3,600 ft ISA). The N-R solver converged to the correct value of $V_{l}$ of 135.8 knot and FLLTOW of $341,437 \mathrm{lbf}$. The AEO TODA net (115\% factored) weight limit is $392,790 \mathrm{lbf}$ and the AEO TORA limit is 396,451 lbf, while the structural takeoff limit (MSTOW) is $410,000 \mathrm{lbf}$. Additionally, it was computed that $V_{l} / V_{R}$ is 0.97 with $V_{R}=140$ and $V_{2}=153$ knots. A slightly longer CWY would result in TORA be more restrictive than TODA. In the case when TOW < FLLTOW for given ASDA and TODA and no reduced-thrust takeoff, a range of $V_{l}$ speeds exists between $V_{G O}$ and $V_{S T P}$ as summarized in Table 1. The abnormal case, $v_{S T P}<v_{G O}$ implies the airplane is performance-wise overloaded and the takeoff is unsafe (see also Figure 9). For 
example, when TOW is $300,000 \mathrm{lb}$, the $V_{G O}$ speed is 113 knots and $V_{S T P}$ speed is 144 knots, while rotation speed is about 132 knots, thus possibly restricting $V_{l}$.

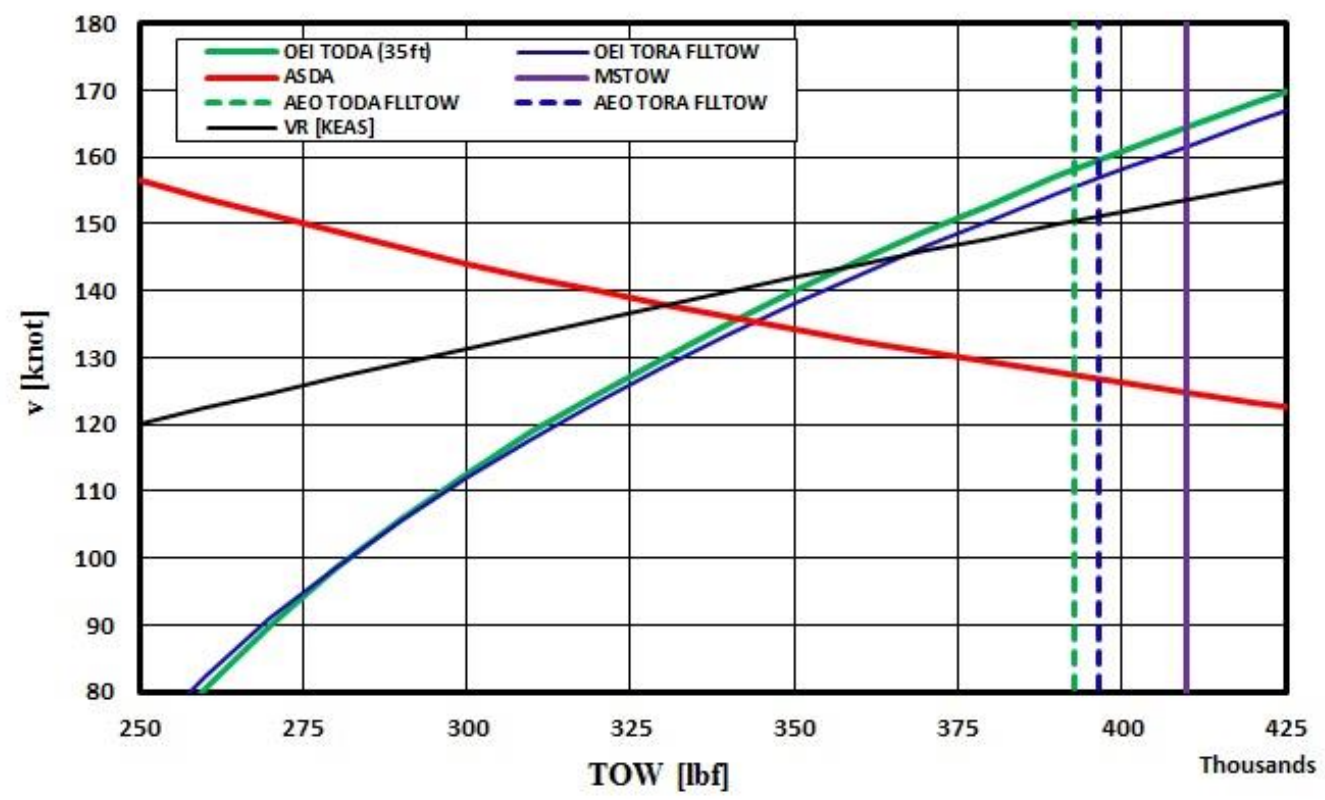

Figure 10. FLLTOW and corresponding $V_{l}$ limited by OEI TODA dry (35 ft) condition with 8,000 ft TORA, $300 \mathrm{ft}$ SWY, $800 \mathrm{ft}$ CWY (UBFL).

Table 1

Minimum and maximum takeoff action speeds when TOW<FLLTOW

\begin{tabular}{cccc}
\hline TOW $[\mathrm{lbf}]$ & $v_{G O}[\mathrm{knot}]$ & $v_{S T P}[\mathrm{knot}]$ & $v_{S T P}-v_{G O}[\mathrm{knot}]$ \\
\hline 300,000 & 112.62 & 144.15 & 31.53 \\
310,000 & 118.84 & 142.00 & 23.15 \\
320,000 & 124.62 & 139.94 & 15.32 \\
330,000 & 130.02 & 137.97 & 7.94 \\
340,000 & 135.11 & 136.08 & 0.97 \\
341,437 & 135.82 & 135.82 & 0.00 \\
345,000 & 137.55 & 135.17 & -2.38 \\
350,000 & 139.92 & 134.27 & -5.65
\end{tabular}


For the BFL case, with no SWY and CWY, as illustrated in Figure 11, we obtain $V_{1}$ of 135.2 knot and OEI TODA-limited FLLTOW of 330,086 lbf at the same no-wind, DA, and dry, hard-surfaced, level runway. The AEO TODA and TORA limits become 374,297 lbf and 396,451 lbf (stays the same) respectively.

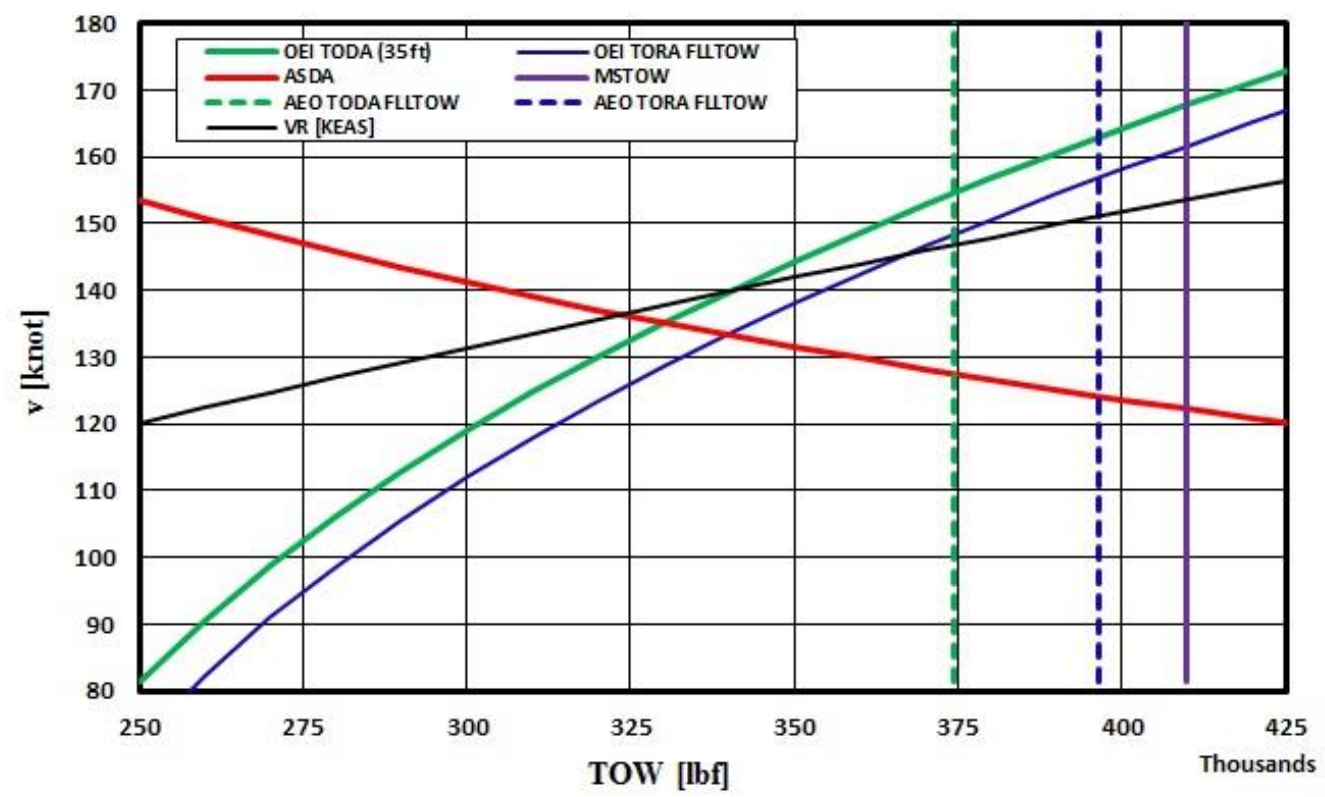

Figure 11. FLLTOW and corresponding $V_{l}$ limited by OEI TODA dry (35 ft) condition with 8,000 ft TORA (BFL), no SWY and CWY.

Clearly, TODR is more limiting than TORR in the absence of CWY. Thus, about 11,400 lbf can be lifted more in UBFL case incorporating relatively short 800-ft CWY and 300-ft SWY. The $V_{l}$ did not change much from the original TORA-only case, as about 2.7 times longer CWY than SWY is added. In this case, $V_{l} / V_{R}$ is 0.98 with $V_{R}=138$ and $V_{2}=150$ knots. On the speed-weight diagram, the ASDA and TODA intersect at a point of FLLTOW and $V_{l}$, but under no circumstances does this imply that ASDA is equal TODA as is often thought. The above solution is given in $T O W-V_{l}$ space. BFL only exists when ASDA=TODA.

UBFC is thus best observed in Figure 12 illustrating UBFL distance-speed graph. Considering wide-body aircraft runway-alignment corrections with 8,000 ft TORA and TODA $600 \mathrm{ft}$ longer than ASDA. Additionally, TODR is still more limiting than TORR. The FLLTOW is thus 345,875 lbf with corresponding $V_{l}$ of 136.79 knots. We have not shown AEO limits, but now the AEO TORR is more limiting at 396,451 lbf than the AEO TODR which is 397,278 lbf. In both above 
cases (UBFL and BFL), the RTOW is limited by unfactored (gross) OEI limits. Clearly, ASDA and TODA differ, but the obtained solution is optimal resulting in maximum takeoff weight (mass) at given $V_{l}$. No consideration and limitations due to $V_{M C G}, V_{M B E}$, and $V_{T I R E}$ is given at this point. They would essentially represent left and right $V_{l}$ boundaries. The minimum $V_{l} / V_{R}$ ratio may be limited by $V_{M C G}$ and the maximum $V_{l} / V_{R}$ ratio may be limited by $V_{M B E}$. Increased TOW will result in ASDR curve moving up and to the left, while TODR curve would move up and to the right.

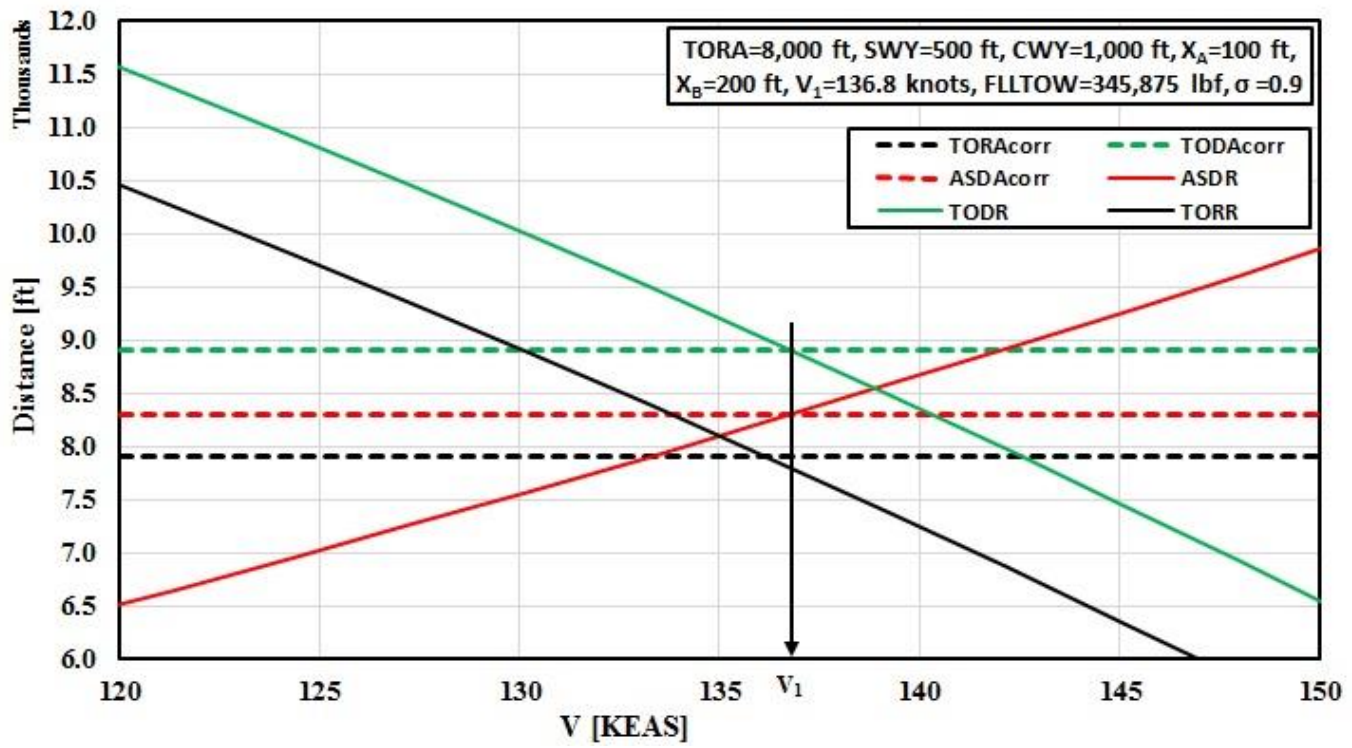

Figure 12. The OEI TODR, TORR, and ASDR distances for 8,000 ft TORA $1,000 \mathrm{ft} \mathrm{CWY}$ and $500 \mathrm{ft} \mathrm{SWY}$ at 3,600 ft ISA.

Using the current airplane model, we have designed several TODR/TORR-ASDR charts for fictitious twin-jet as a function of air density with TOW and $V_{l} / V_{R}$ as parameters. Of course, this applies only to no-wind, dry, level, and hard-surfaced (paved) runway. The SL ISA performance diagram (TODR/TORR-ASDR) is shown in Figure 13. The balanced field conditions occur for $V_{l} / V_{R}$ speed ratios in the range from about 0.96 (lower weights) to 0.975 (higher weights). When $V_{l} / V_{R}$ is equal to one, $V_{l}$ is limited by $V_{R}$. Unlimited number of BFCs and UBFCs are thus possible. At constant weight, increasing $V_{l} / V_{R}$ results in decreased TODR and increased ASDR. The vertical projection (ASDR is constant) of difference between the corresponding TODR and TORR (for the same TOW), represents $1 / 2$ of the airborne distance. Moving along the constant $V_{l} / V_{R}$ and increasing TOWs causes increase in both ASDR and 
TODR/TORR. However, at lower $V_{l} / V_{R}$, the increase in TODR is steeper than in ASDR (slope is shallower than for BFC).

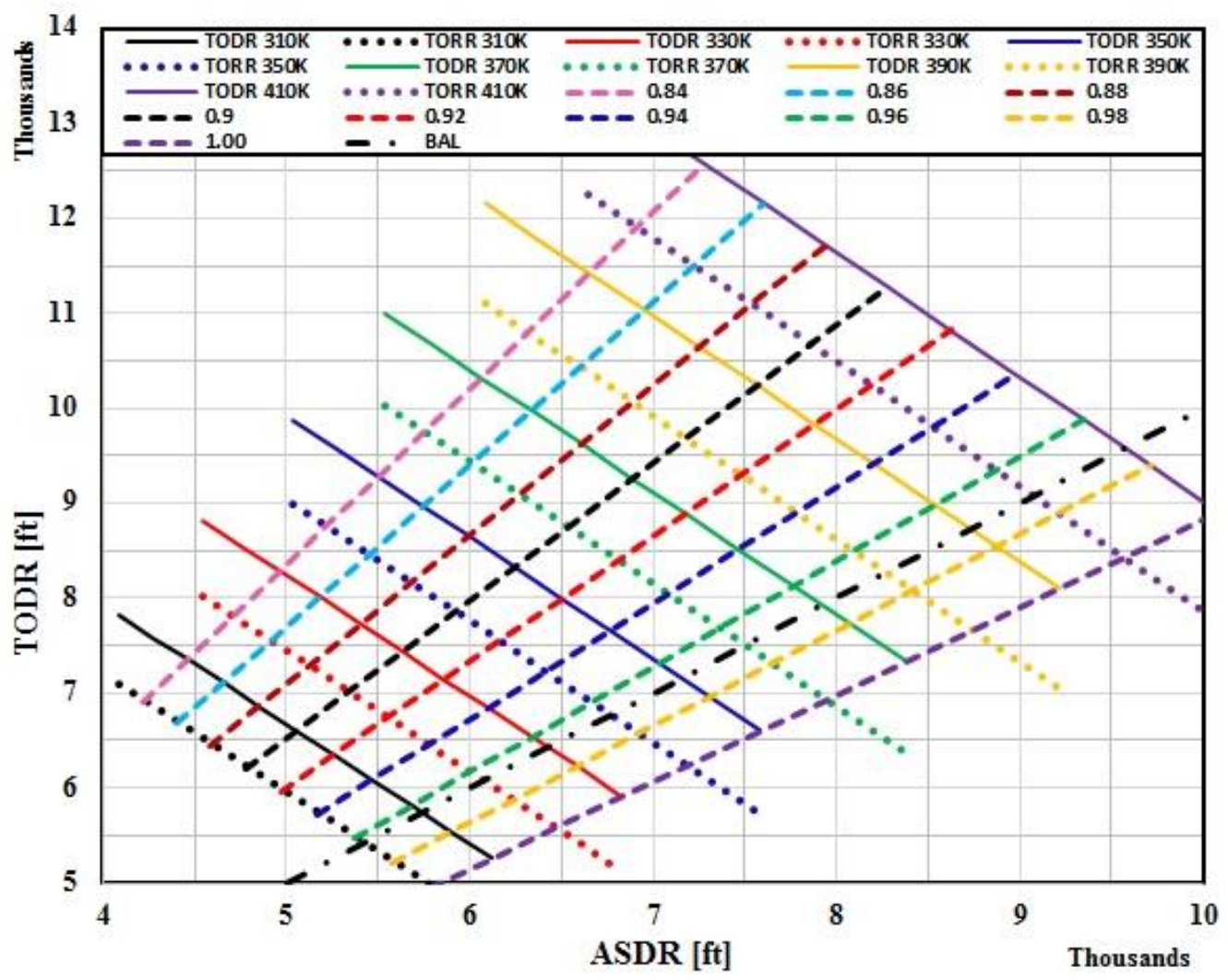

Figure 13. OEI TODR and TORR versus ASDR for different TOWs and $V_{l} / V_{R}$ ratios at SL ISA conditions $(\sigma=1.0)$.

Going to lower air density (DA is 3,600 ft) is illustrated by computations summarized in Figure 14 . The BFC is now very close to $V_{l}=V_{R}$ limit (around 0.99). The low-speed $V_{l} / V_{R}$ ratio results in even steeper slopes and TODR/TORR naturally increases even faster than ASDR. At low $V_{l} / V_{R}$, more time is spent in OEI acceleration and the engine thrust decreases significantly with the lower air density resulting in TODR/TORR being significantly longer than ASDR. For example, lifting $390 \mathrm{~K} \mathrm{lbf}$ at $V_{l} / V_{R}=0.88$ (possibly $V_{M C G}$ limited) ASDR becomes about $8,400 \mathrm{ft}$, TODR is $14,000 \mathrm{ft}$ and TORR is $12,500 \mathrm{ft}$. At the same TOW and $V_{l} / V_{R}=0.96$, TODR becomes $11,600 \mathrm{ft}$, ASDR is almost $10,000 \mathrm{ft}$, and TORR is $10,300 \mathrm{ft}$. BFCs are still possible, but the aircraft is on the brink of becoming $V_{R}$ limited. 


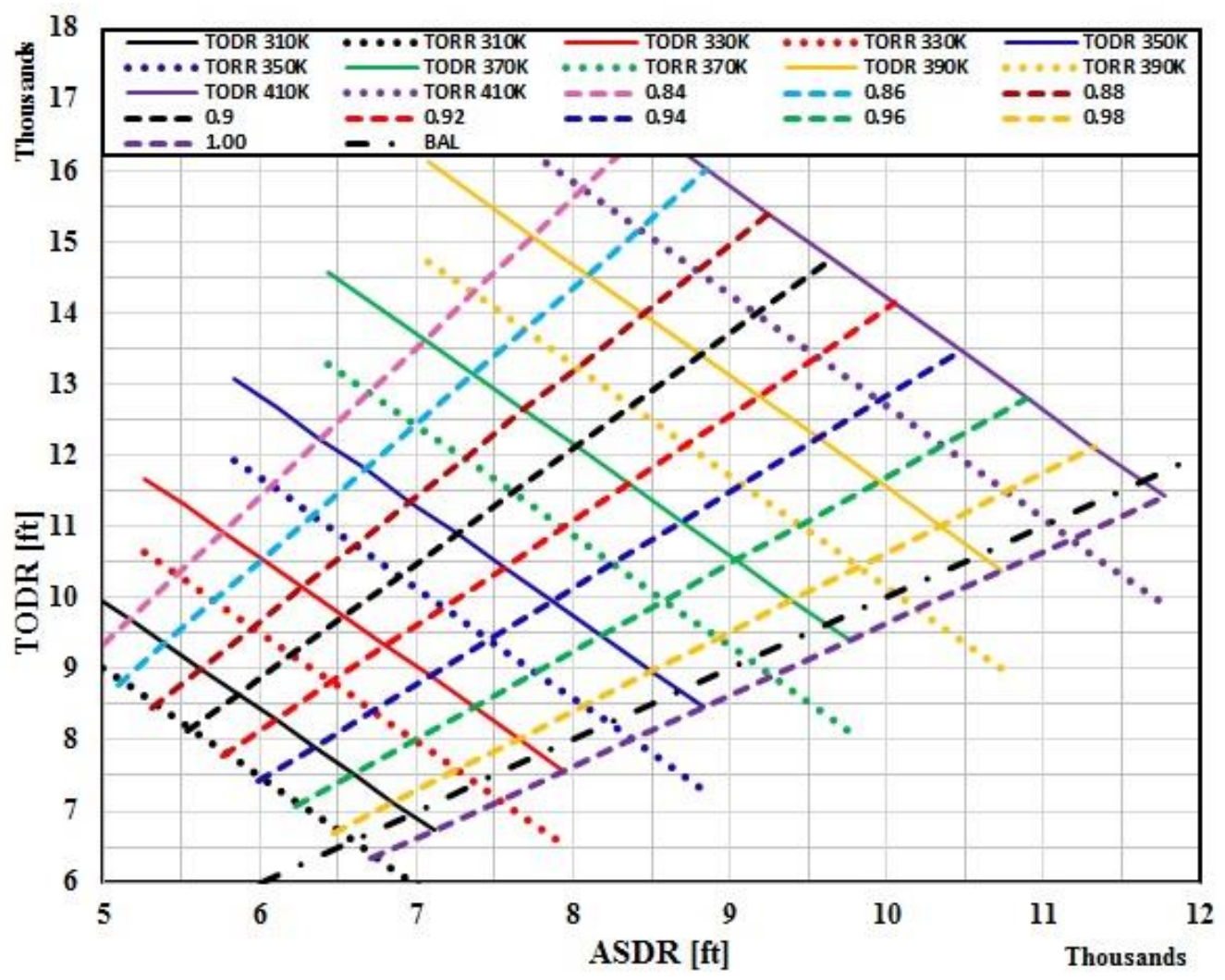

Figure 14. OEI TODR and TORR versus ASDR for different TOWs and $V_{l} / V_{R}$ ratios at about $3,600 \mathrm{ft}$ ISA conditions $(\sigma=0.9)$.

Taking off from about 7,400 ft ISA airfield, causes significant decrease in TOW for the same runway sizes as illustrated in Figure 15. TODR starts increasing even steeper than ASDR. All TOWs result in unbalanced conditions and are limited by rotation speeds $V_{l}=V_{R}$. The slopes of constant speed-ratios become very steep at low $V_{l} / V_{R}$, requiring excessive TORAs and TODAs.

Runway analysis charts or RTOW graphs/charts (Swatton, 2008) can be now constructed for desired airport/runway and aircraft airframe/powerplant combination. The base conditions are for no-wind, dry, hard-surfaced, level runway. Corrections can then be applied for wind (HW, TW, XW), effective runway longitudinal slope, non-dry runway surface conditions (wet, contaminated), anti-skid op/inop, available thrust (maximum/flexible, AC on/off, TMC/PMC on/off, etc.), anti-ice on/off, and environmental/atmospheric conditions (temperature, pressure, humidity). Some of the corrections were already discussed All relevant aerodynamic and mechanical limits/constraints can 
be included to define RTOW under given conditions. The range of $V_{l}$ speeds exists when aircraft is not FLLTOW limited. Reduced and/or derated thrust takeoffs are common in such cases (Daidzic, 2012a, 2012b).

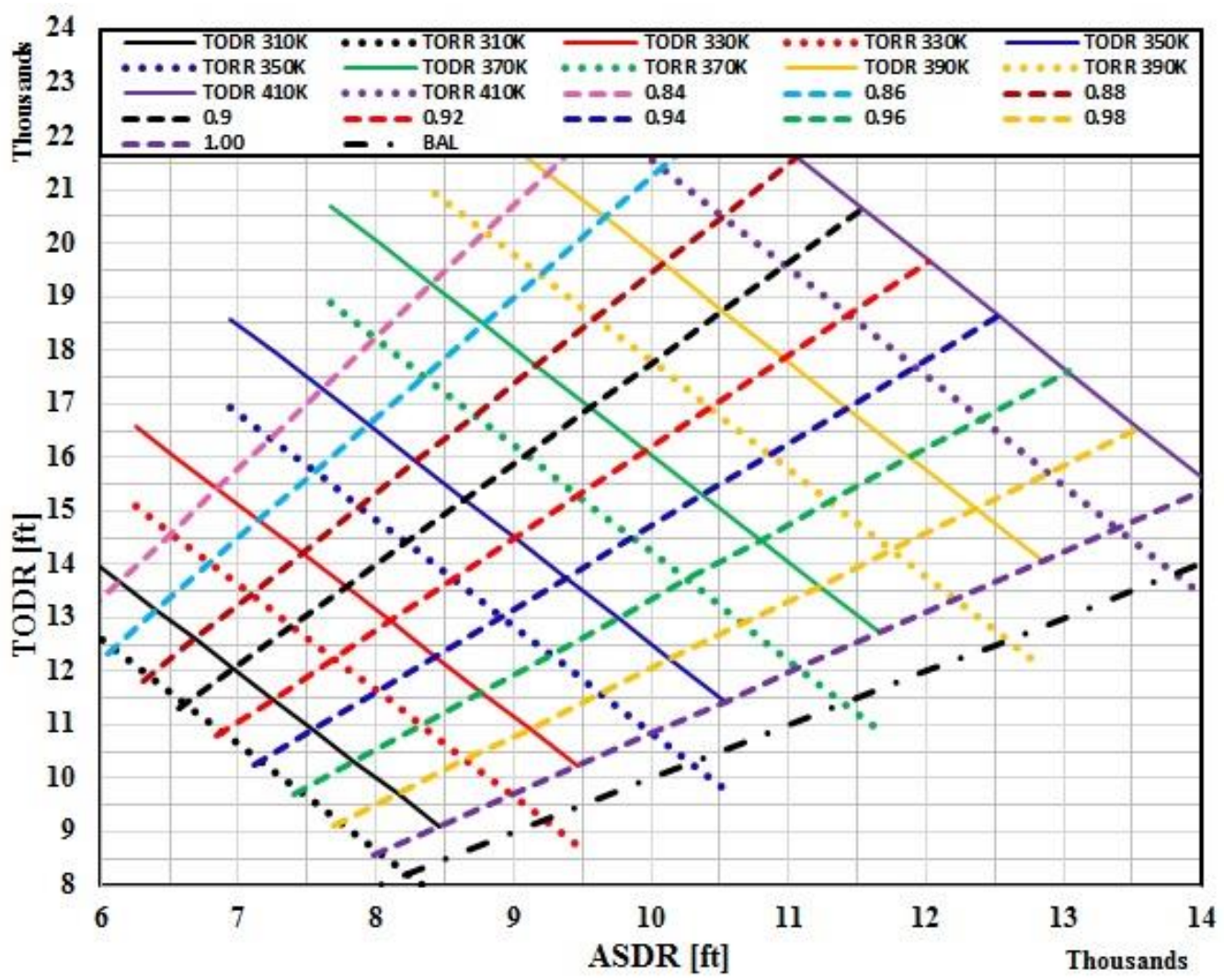

Figure 15. TODR and TORR versus ASDR for different TOWs and $V_{l} / V_{R}$ ratios at about $7,400 \mathrm{ft}$ ISA conditions $(\sigma=0.8)$.

Perhaps the most important result of this study is depicted in Figure 16. It illustrates the increase of FLLTOW by adding runway extensions in various proportions. The FLLTOW for the basic dry and level ASDA=TODA=BFL is $330,086 \mathrm{lbf}$ with $V_{l}$ equal to 135.2 knots. The $V_{l} / V_{R}$ ratio is 0.98 and $V_{2}$ is 150.4 knots (shown in Figure 11). Adding up to 2,000 $\mathrm{ft}$ of CWY and/or SWY to the basic 8,000-ft TORA (up to 25\% increase) is simulated. Adding SWY alone will increase FLLTOW, but only if $V_{l}$ is higher. On the other hand, adding CWY increases FLLTOW at the expense of lower $V_{l}$. Thus, $\left(\partial v_{1} / \partial S W Y\right)_{W}>0$, and $\left(\partial v_{1} / \partial C W Y\right)_{W}<0$. See also Figure 9 for illustration and graphical explanation. Adding both SWY and CWY at the same rate (BFL) will increase FLLTOW 
significantly, while exact change in $V_{l}$ will have to be determined, but is often somewhat increasing. However, $V_{l}$ increase is not as dramatic as is in the case of adding SWY only. Thus, we could state quite generally that CWY reduces and SWY increases $V_{l}$. All the presented results apply only to a specific airplane, nowind, dry and level runway, and given air density.

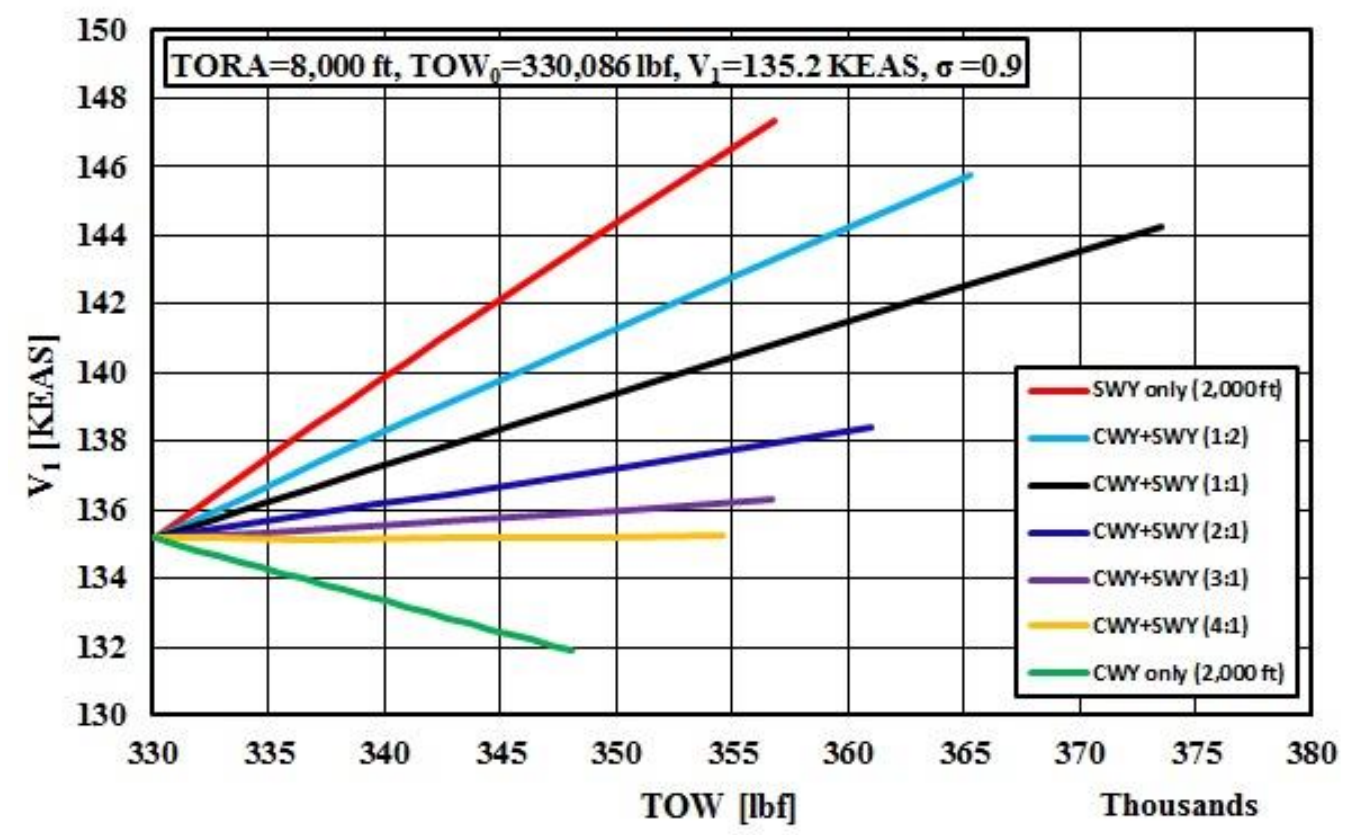

Figure 16. The effect of up to 2,000 ft runway extensions (CWY and/or SWY) on $V_{l}$ change and FLLTOW increase at about 3,600 ft ISA.

Also interesting is that weight/speed increments are practically linear. For example, adding 2,000 ft of SWY to 8,000 TORA, increased TOW by 26,764 lbf $(8.1 \%)$ at the expense of $V_{l}$ increase by 12.2 knots. Assuming linear trend each foot of SWY increases FLLTOW by $13.4 \mathrm{lbf}$. For this case $V_{l}$ increased by about $0.61 \mathrm{knot} / 100 \mathrm{ft}$. In the case when SWY and CWY were added in equal amounts (BFL or 1:1 ratio) of 2,000 ft, then each foot of SWY and CWY together added $21.73 \mathrm{lbf}\left(13.2 \%\right.$ increase) to basic TOW of 330,085.75 lbf. At the same time $V_{l}$ increased by 9.1 knots or +0.455 knots for each $100 \mathrm{ft}$ of combined SWY and CWY. Adding up to $2,000 \mathrm{ft}$ of CWY only, increased FLLTOW merely by $17,981 \mathrm{lbf}(5.4 \%)$, while $V_{l}$ decreased 3.26 knots, which is $-0.163 \mathrm{knots} / 100 \mathrm{ft}$. Another problem with long CWY is that at some critical length, TORR will constrain takeoff rather than TODR, and any CWY longer than the critical becomes unusable for takeoff performance scheduling. Adding up to 2,000 ft of CWY and 1,000 ft SWY (2:1 ratio) increased TOW by about 31,000 lbf (9.4\%) at 
a cost of 3 knot faster $V_{l}$. The $V_{l} / V_{R}$ is 0.96 and $V_{2}$ is 157 knots. Using wet runway $V_{\text {lwet }}$ of 126 knots and lowering $\mathrm{SH}$ to $15 \mathrm{ft}$ resulted in maximum liftoff weight of $340,800 \mathrm{lbf}, V_{R} / V_{2}$ of $140 / 153$ knots with the same 2,000/1,000 $\mathrm{ft}$ $\mathrm{CWY} / \mathrm{SWY}$ ratio. At the CWY/SWY ratio of $4: 1$, the $V_{1}$ speed essentially stays unchanged, while FLLTOW increased by almost 25,000 lbf (7.5\%). The nondimensional changes in $v_{1}^{*}$ and TOW* for specified SWY and CWY lengths are presented in Figure 17.

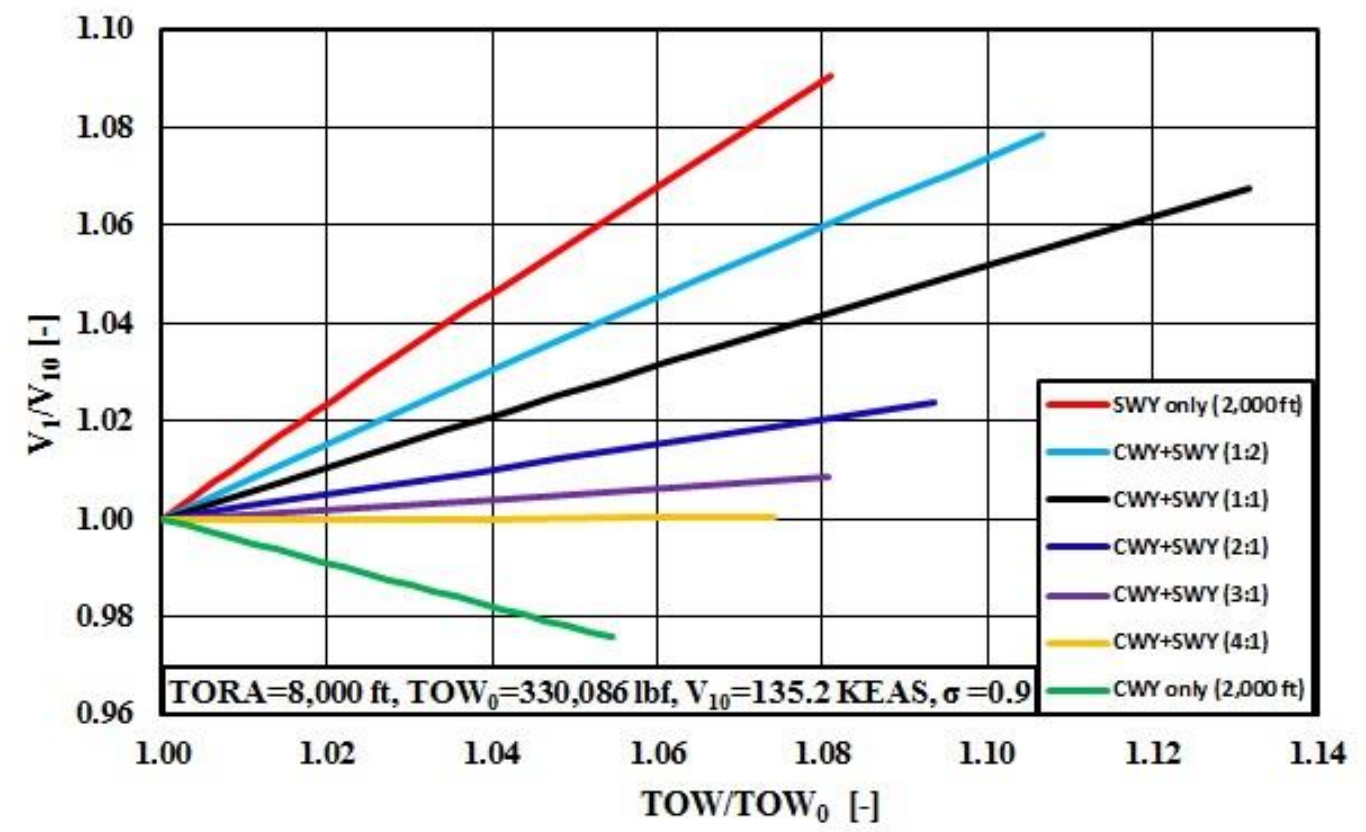

Figure 17. The effect of up to 2,000 ft runway extensions (CWY and/or SWY) on dimensionless $V_{l}$ and TOW changes at about 3,600 ft ISA.

Interestingly, the combined balanced SWY+CWY (SCWY) extension length, results in general triangle problem, which approximately obeys the Cosine Law (Spiegel and Liu, 1999). The change in $V_{l}$ and TOW for combined SCWY length can be found from adding respective projections of SWY- and CWY-only cases. The angle is defined by the respective first-order derivatives with respect to runway extensions at constant weight. The last graphical result is shown in Figure 18. It depicts the same result given in Figures 16 and 17, but this time the increase in TOW is given directly as a function of runway extensions for various combinations of CWYs and SWYs. It is assumed again that TORA is not limiting TOW. Interestingly, up to 2,000 ft of SWY only (with associated 12.2 knots 
increase in $V_{l}$ ) increased TOW at virtually the same rate as CWY/SWY ratio of three (up to 2,000 $\mathrm{ft} \mathrm{CWY}$ and up to $667 \mathrm{ft}$ of SWY) runway extension.

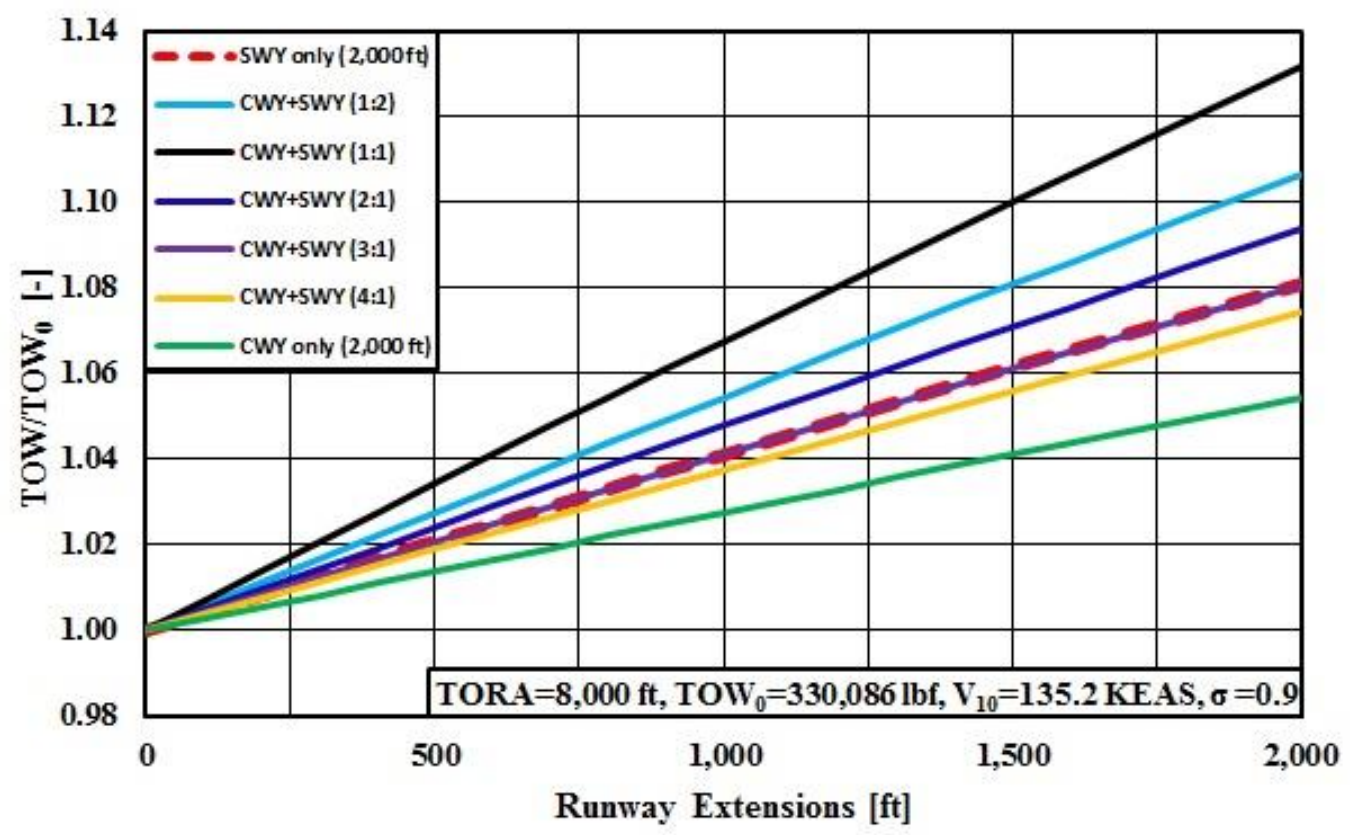

Figure 18. The effect of up to 2,000 ft runway extensions (CWY and/or SWY) on dimensionless $V_{l}$ and TOW changes at about 3,600 ft ISA.

The total cost of modern runways that support operations of wide-body super-jumbo T-category jets (e.g., A380) may be around $\$ 1,000 / \mathrm{ft}^{2}$ (with the SD of $\$ 100 / \mathrm{ft}^{2}$ ). Thus a modern $9,000 \times 150 \mathrm{ft}$ runway with land purchase and all associated infrastructure may cost around US\$ 1.35 billion today and will take several years to build. On the other hand, the cost of CWY (500 ft or $150 \mathrm{~m}$ wide) is estimated at $\$ 20 / \mathrm{ft}^{2}$, while the SWY may cost about $\$ 250 / \mathrm{ft}^{2}$. Thus a $1,000 \mathrm{x}$ $500 \mathrm{ft}$ CWY would today cost US\$ 10 million. A $500 \times 150 \mathrm{ft}$ SWY to support heaviest jets may cost today $\$ 18,750,000$. An economic analysis of using runway extensions could now be conducted to facilitate the estimation of the most efficient combinations and lengths of TORA's, SWYs and CWYs.

Crucial parameters in this energy analysis are: $E=\sigma \cdot A S D A_{\text {corr }}$, $U_{1}=\sigma \cdot T O D A_{c o r r}$, and $U_{2}=\sigma \cdot T O R A_{\text {corr }}$. These density-dependent values define volumes of runway energy reservoirs that can be used in energy conversion during airplane takeoffs. For example, during AS maneuver, the runway first provides energy exchange for an airplane to gain kinetic energy and then subsequently 
energy absorption during braking efforts. In the case of AG maneuvers (TORR or TODR), the runway provides necessary space for the airplane to achieve minimum required and safe total energies for, both, the AEO and/or OEI cases under various environmental conditions. Since, airplanes need the same operational EAS/CAS speed at given weight, the runway potential energy reservoirs are being significantly depleted at lower air densities (higher DA).

We tried to strike a balance between the complexity and the fidelity/accuracy. The results presented are not far from the certified takeoff data for airplanes in the same category as tested on the fictitious aircraft here. Future improvements in the presented total-energy model will include more accurate modeling of forces. Additionally, the $V_{M C G}, V_{M B E}$ and $V_{T I R E}$ speed limits will be modeled and showcased in subsequent publications. The full non-dimensional takeoff equations, which may also include full steady-wind effects will be presented in a future contribution. Based on a limited validation study, we believe that TPT is currently accurate within $3 \%$ in the entire takeoff envelope for a given airplane model. However, this is work in progress and we have already identified parts where further improvements will be made. Ultimately, the goal is to achieve overall accuracy and fidelity of less than $0.5 \%$ compared to flight-test data for particular T-category airplane make and models. Nevertheless, using the totalenergy takeoff models can be made more accurate and reliable if data from actual flight tests are used to calibrate TPT analytic model. Furthermore, this totalenergy based model offers better understanding of takeoff physics and identify effects of individual variables. TPT is very flexible and can accommodate any regulatory changes, any conventional-takeoff airplane models, runways and environmental conditions. The model can be coded in any high-level computer language. Future contributions will focus on wet and contaminated runways and, in more detail, the effects of air density, wind, and runway slope with CWY/SWY extensions.

To reiterate, the main motivation of this work is to develop a relatively simple, yet reasonably accurate and reliable, model that can serve as a powerful tool in deeper understanding of complicated takeoff dynamics of FAR/CS 25 airplanes used in commercial services. A variety of calculations for differing runway layouts with or without runway extensions, aircraft, and environmental conditions can be performed. Particular emphasis of this work was the effect oof SWYs and/or CWYs on $V_{l}$ and FLLTOW. This could also be very helpful in designing new airports and runways and the feasibility and economic analysis of runways and extensions. Additionally, the goal was to dispel some common misconceptions on FAR/CS 25 takeoff physics. At the end, the problem of 
determining the maximum takeoff weight is a problem of nonlinear constrained optimization with many constraints in the form of equalities and inequalities.

\section{Conclusions}

A physics-based total-energy airplane takeoff performance model has been developed. Average accelerating and decelerating forces during ground and airborne takeoff segments have been modeled. Hence, accurate time histories cannot be predicted, but only the end states. Current FAA CFR and EASA CS takeoff regulations have been implemented. The main model consists of three sub-models: runway model, aircraft model, and atmospheric model. A fictitious wide-body T-category airplane has been used for simulations. A Newton-Raphson nonlinear equation solver was used to calculate performance-limited takeoff weight and the associated takeoff decision/action speed. All other takeoff speeds were extracted as well. A simplified mathematical analysis of the nonlinear constrained optimization problem showed that estimated weights and speeds are indeed optimums. Special emphasis was given to UBFL takeoffs and the individual and combined effect of CWYs and/or SWYs was explored. The length of CWY was increased until TORA becomes limiting designating the critical CWY length. As expected, the maximum liftoff weights are obtained when both CWYs and SWYs are used together, which is also the most expensive solution. The use of CWYs only, although being the cheapest solution increased takeoff weight by smallest amount. For given DA at CWY/SWY ratio of about 4:1, the $V_{l}$ stays essentially unchanged, while TOW increases significantly. Runway analysis charts or RTOW charts could be now readily designed for an arbitrary airframe/powerplant combination, airport/runway with declared distances, runway surface quality and grade, atmospheric conditions including wind and air density, and other limitations. In future developments, the takeoff performance tool will include various improvements. Although the presented TPT cannot replace demonstrated and measured takeoff data it can serve as a flight-test expansion model for various airplane types if measured performance is used to augment and calibrate it. TPT nevertheless serves as a useful tool in understanding takeoff physics and the many limitations that often lead to confusing nonlinear interaction

of many parameters. Presented model is flexible and can accommodate any future regulatory changes and can be also used in various technical and economic optimization studies. 


\section{Author Bio}

Dr. Nihad E. Daidzic is president of AAR Aerospace Consulting, L.L.C. He is also a full professor of Aviation, adjunct professor of Mechanical Engineering, and research graduate faculty at Minnesota State University, Mankato. His Ph.D. is in fluid mechanics and Sc.D. in mechanical engineering. He was formerly a research associate in applied physics at the Center for Microgravity Research and Applications at Vanderbilt University and a staff scientist at the National Center for Microgravity Research and the National Center for Space Exploration and Research at NASA Glenn Research Center in Cleveland, OH. He has also held various faculty appointments at Vanderbilt University, University of Kansas, and Kent State University. His current research interest is in theoretical, experimental, and computational fluid dynamics, micro- and nano-fluidics, aircraft stability, control, and performance, mechanics of flight, piloting techniques, and aerospace propulsion. Dr. Daidzic is ATP and "Gold Seal" CFII/MEI/CFIG with flight experience in airplanes, helicopters, and gliders. 


\section{References}

Airbus. (2002). Getting to grips with aircraft performance (Flight operations support and line assistance). Blagnac, France: Author.

Allerton, D. (2009). Principles of flight simulation. Chichester, West Sussex, UK: John Wiley \& Sons.

Anderson, J. D. Jr. (1999). Aircraft performance and design. New York, NY: McGraw-Hill.

Asselin, M. (1997). An introduction to aircraft performance. Reston, VA: American Institute for Aeronautics and Astronautics (AIAA).

Australian Transport Safety Board. (ATSB). (2011). Take-off performance calculation and entry errors: A global perspective (AR-2009-052). Retrieved from www.atsb.gov.au

Ayres, F., Jr., \& Mendelson,E. (2009). Calculus (5th ed.). New York, NY: McGraw-Hill.

Bays, L. V. \& Halpin, K. E. (2014). Improved safety and capability via direct computation of takeoff and landing performance data, 14th AIAA Aviation Technology, Integration, and Operations Conference, AIAA Aviation, 16-20 June 2014, Atlanta, GA. DOI: 10.2514/6.2014-2154

Boeing Commercial Airplanes. (2005). 767 Airplane characteristics for airport planning (D6-58328). Seattle, WA: Author.

Carnahan, B., Luther, H. A., \& Wilkes, J. O. (1969). Applied numerical methods. New York, NY: John Wiley \& Sons.

Chapra, S. C., \& Canale, R. P. (2006). Numerical methods for engineers (5th ed.). Boston, MA: McGraw-Hill.

Chiles, P. (2007, July). Planning the departure: Takeoff performance myths and methods. AeroSafety World, 2(7), 26-32.

Civil Aviation Authority. (UK CAA) (2006). CAP 698 CAA JAR-FCL examinations: Aeroplane performance manual (3rd ed.). West Sussex, UK: Author. 
Daidzic, N. E., \& Shrestha, J. (2008). Airplane landing performance on contaminated runways in adverse conditions, Journal of Aircraft, 45(6), 2131-2144. DOI: $10.2514 / 1.38056$

Daidzic, N.E. (2011). Regional airline operations on contaminated runways, WATS 2011, World Aviation Training Conference, Rosen Shingle Creek Resort, Paper RATS 6.3, 19-21 April 2011, Orlando, FL.

Daidzic, N. E. (2012a, March). FADEC advances allow better Engine Performance, Professional Pilot, 46(3), 78-82.

Daidzic, N. E. (2012b, September). Jet engine thrust ratings, Professional Pilot, 46(9), 92-96.

Daidzic, N. E. (2013a, August). T/O overruns and veer-offs on slippery runways with crosswinds, Professional Pilot, 47(8), 54-58.

Daidzic, N. E. (2013b, November). Aircraft tests arrive at numbers pilots need for safe operations, Professional Pilot, 47(11), 100-104.

Daidzic, N. E. (2014a). A total-energy based model of airplane overspeed takeoffs, International Journal of Aviation Aeronautics Aerospace (IJAAA), 1(3), 1-25. DOI: 10.15394/ijaaa.2015.1016

Daidzic, N. E. (2014b). Improving airplane touchdown control by utilizing the adverse elevator effect, International Journal of Aviation Aeronautics Aerospace (IJAAA), 1(3), 1-25. DOI: 10.15394/ijaaa.2015.1032

Daidzic, N. E. (2015a). Efficient general computational method for estimation of standard atmosphere parameters, International Journal of Aviation Aeronautics, and Aerospace, 2(1), 1-35. DOI: 10.15394/ijaaa.2015.1053

Daidzic, N. E. (2015b). Global optimized isothermal and nonlinear models of Earth's standard atmosphere, International Journal of Aviation Aeronautics, and Aerospace, 2(3), 1-42. DOI: 10.15394/ijaaa.2015.1064

Daidzic, N. E. (2016a). General solution of the wind triangle problem and the critical tailwind angle, The International Journal of Aviation Sciences (IJAS), 1(1), 57-93. 
Daidzic, N. E. (2016b). Determination of rejected landing roll runway point-ofno-return and go-around in transport category airplanes, International Journal of Aviation Aeronautics Aerospace (IJAAA), 3(1), 1-31. DOI: 10.15394/ijaaa.2015.1110

Daidzic, N. E. (2016c). Estimation of performance airspeeds for high-bypass turbofans equipped transport-category airplanes, Journal of Aviation Technology and Engineering (JATE), 5(2), pp. 1-24. DOI: 10.7771/21596670.1122

Daidzic, N.E. (2016d). Utilization of TRSS to assist pilot's decision making process during critical runway operations, WATS 2016, World Aviation Training Conference, Rosen Shingle Creek Resort, Paper WATS 5.3, April 19-21, 2016, Orlando, FL.

Davis, D. P. (1971). Handling the big jets (3rd ed.). London, UK: Civil Aviation Authority.

Demidovich, B. P., \& Maron, I. A. (1987). Computational mathematics. Moscow, Russia: MIR.

Durbin, E. J., \& Perkins, C. D. (eds.) (1962). Flight test manual (AGARD Volume 1, Chapter 8, 2nd ed.). Oxford, UK: Pergamon Press.

Eshelby, M. E. (2000). Aircraft performance: Theory and practice. Boston, MA: Elsevier.

European Aviation Safety Agency. (EASA) (2007). Certification specifications for large aeroplanes CS-25 (Amendment 3, 19 September). Cologne, Germany: Author.

Filippone, A. (2008). Comprehensive analysis of transport aircraft flight performance, Progress Aerospace Sciences, 44(3), 192-236. DOI: 10.1016/j.paerosci.2007.10.005

Filippone, A. (2012). Advanced aircraft flight performance. New York, NY: Cambridge University Press.

Hale, F. J. (1984). Introduction to aircraft performance, selection, and design. New York, NY: John Wiley \& Sons. 
Holt, M. J., \& Poynor, P. J. (2006). Air carrier operations. Newcastle, WA: Aviation Supplies \& Academics.

Hurt, H. H. (1965). Aerodynamics for naval aviators. Renton, WA: Aviation Supplies \& Academics.

International Civil Aviation Organization. (ICAO) (2006). Aerodrome design manual (Doc 9157 AN/901, 3rd ed.). Montreal, CA: Author.

Jeppesen. (2007). Performance. (JAA ATPL Training, Edition 2, JA 310109-002, Book 9, JAR Ref. 032). Neu-Isenburg, Germany: Atlantic Flight Training, Ltd., Sanderson Training products, Jeppesen GmbH.

de Lemos Viana, J. P. R. (2011). Takeoff and landing performance optimization: Development of a computational methodology (Master's thesis, Instituto Superior Tecnico, Universidade Tecnica de Lisboa, Lisbon, Portugal). Retrieved from https://fenix.tecnico.ulisboa.pt/

Loftin, Jr. L. K. (1980). Subsonic aircraft: Evolution and the matching of size to performance (NASA RP 1060). Washington, DC: National Aeronautics and Space Administration.

Lowery, J. (2001). Professional pilot (2nd ed.). Ames, IA: Iowa University Press.

Luenberger, D. G. (1984). Linear and nonlinear programing (2nd ed.). Reading, MA: Addison-Wesley.

Mair, W. A., \& Birdsall, D. L. (1992). Aircraft performance. Cambridge, UK: Cambridge University Press.

McCormick, B. W. (1995). Aerodynamics, aeronautics and flight mechanics (2nd ed.). New York, NY: John Wiley \& Sons.

Minoux, M. (1986). Mathematical programing; Theory and algorithms. Chichester, West Sussex, UK: John Wiley \& Sons.

Nuic, A., Poinsot, C., Iagaru, M.-G., Gallo, E., Navarro, F. A., \& Querejeta, C. (2005). Advanced aircraft performance modeling for ATM: Enhancements to the BADA model, 24 ${ }^{\text {th }}$ Digital Avionics System Conference, October 30-November 3, Washington D.C. DOI: 10.1109/DASC.2005.1563320 
Ohme, P. (2009). A Model-based approach to aircraft takeoff and landing performance assessment, AIAA Atmospheric Flight Mechanics Conference, Guidance, Navigation, and Control, 10-13 August 2009, Chicago, Illinois. DOI: 10.2514/6.2009-6154

Ojha, S. K. (1995). Flight performance of aircraft. Washington, DC: American Institute for Aeronautics and Astronautics (AIAA).

Padilla, C. E. (1996). Optimizing jet transport efficiency: Performance, operations, and economics. New York, NY: McGraw-Hill.

Perry, D. H. (1967). An analysis of some major factors involved in normal takeoff performance (C.P. No. 1034), UK Ministry of Technology, Aeronautical Research Council, UK: Her Majesty's Stationery Office.

Pierre, D. A. (1986). Optimization theory with applications. Mineola, NY: Dover.

Press, W. H, Teulkolsky, S. A., Vetterling, W. T., \& Flannery, B. P. (1992). Numerical recipes in FORTRAN: The art of scientific computing (2nd ed.). Cambridge, UK: University Press.

Roskam, J., \& Lan, C. T. (1997). Airplane aerodynamics and performance, Lawrence, KS: DAR Corporation.

Saarlas, M. (2007). Aircraft performance. Hoboken, NJ: John Wiley \& Sons.

Simos, D. (2006). PIANO: A tool for preliminary design, competitor evaluation, performance analysis. In ICAO Committee on Aviation Environmental Protection (CAEP) working group 2 meeting, May 2006, Rome, Italy.

Smith, L. P. (1953). Mathematical methods for scientists and engineers. New York, NY: Prentice-Hall, Inc.

Smith, M. A. (1953, January 16). Stopways and clearways. Flight and Aircraft Engineer, LXIII(2295), 67-68.

Spiegel, M. R., \& Liu, J. (1999). Mathematical handbook of formulas and tables (2nd ed.). New York, NY: McGraw-Hill.

Swatton, P. J. (2008). Aircraft performance: Theory and practice for pilots (2nd ed.). Chichester, UK: John Wiley \& Sons. 
Torenbeek, E. (2013). Advanced aircraft design: Conceptual design, technology and optimization of subsonic civil airplanes. Chichester, West Sussex, UK: John Wiley \& Sons.

US Department of Transportation, Federal Aviation Administration. (1994). Takeoff safety training aid (AC 120-62). Washington, DC: Author.

US Department of Transportation, Federal Aviation Administration. (2005). Runway length requirements for airport design (Advisory Circular AC 150/5325-4B, 7/1/2005). Washington, DC: Author.

US Department of Transportation, Federal Aviation Administration. (2011). Flight test guide for certification of transport category airplanes (Advisory Circular AC 25-7C, 10/16/2012). Washington, DC: Author.

US Department of Transportation, Federal Aviation Administration. (2013). Part 25, Airworthiness standards: Transport category airplanes. Washington, DC: Author.

US Department of Transportation, Federal Aviation Administration. (2014a). Airport design (AC 150/5300-13A). Washington, DC: Author.

US Department of Transportation, Federal Aviation Administration. (2014b). Part 121, Operating requirements: Domestic, flag, and supplemental operations. Washington, DC: Author.

Vinh, N. X. (1993). Flight mechanics of high-performance aircraft. Cambridge, UK: Cambridge University Press.

Wagenmakers, J. (1991). Aircraft performance engineering. New York, NY: Prentice Hall.

Wasiuk, D. K., Lowenburg, M. H., \& Shallcross, D. E. (2015). An aircraft performance model implementation for the estimation of global and regional commercial aviation fuel burn and emissions. Transportation Research Part D, 35, 142-159. DOI: 10.1016/j.trd.2014.11.022

Webb, J., \& Walker, B. (2004). Fly the wing (3rd ed.). Ames, IA: Blackwell.

Zontul, M. (2013). Rule based aircraft performance systems. International Journal of Soft Computing and Engineering (IJSCE), 3(4), 61-66. 


\section{Appendix A}

Modeling of dominant forces during takeoff

If the accelerated takeoff roll is restricted to 1-D straight-line motion only, the distance and the time covered to accelerate between two groundspeeds is (Vinh, 1993):

$s=\int_{v_{1}}^{v_{2}} \frac{v d v}{a(v)}=\frac{v_{2}^{2}-v_{1}^{2}}{2 \bar{a}} \quad t=\int_{v_{1}}^{v_{2}} \frac{d v}{a(v)}=\frac{v_{2}-v_{1}}{\bar{a}}$

The instantaneous acceleration using EAS can be modeled using $2^{\text {nd }}$-order polynomial (Vinh, 1993):

$$
a(v)=A+B \cdot v+C \cdot v^{2}
$$

where the instantaneous coefficients are,

$$
\begin{aligned}
& A=\frac{g}{W} \cdot\left\{n_{e} \cdot T S P \cdot T_{S L} \cdot \sigma^{m} \cdot-\left[\mu_{r}(v)+\phi(v)\right] \cdot W\right\} \\
& B=\frac{g}{W} \cdot n_{e} \cdot T S P \cdot T_{S L} \cdot \sigma^{m} \cdot \frac{c_{1}}{\sqrt{\sigma}} \\
& C=\frac{g}{W} \cdot\left\{n_{e} \cdot T S P \cdot T_{S L} \cdot \sigma^{m} \cdot \frac{c_{2}}{\sigma}-\frac{1}{2} \rho_{S L} S_{r e f}\left[C_{D}-\mu_{r}(v) \cdot C_{L}\right]\right\}
\end{aligned}
$$

Generally, the coefficients $A$ and $C$ are speed-dependent. They can be made constant if average values of selected coefficients are used. Runway upslope defines positive, while downslope defines negative local gradient $\phi$. The total drag coefficient accounting for ground-effect (Anderson, 1999) and in a given airplane configuration (landing gear, flaps/slats, etc.) is approximately:

$$
C_{D}=C_{D, 0}+\psi(h / b) \cdot K \cdot C_{L}^{2} \quad \psi(h / b)=\left\{\begin{array}{cc}
1 & h / b>1 \\
\frac{(16 \cdot h / b)^{2}}{1+(16 \cdot h / b)^{2}} & h / b \leq 1
\end{array}\right\}
$$

Propulsive thrust of modern turbofans is a function of actual air density and true airspeed for a given bypass-ratio and other design characteristics. Thrust from a single engine can be written as (Daidzic, 2016c): 


$$
\begin{aligned}
& T_{1 e}\left(\sigma, v_{T A S}, T S P\right)=T S P \cdot T_{S L} \cdot \sigma^{m} \cdot\left(1+c_{1} \cdot v_{T A S}+c_{2} \cdot v_{T A S}^{2}\right) \\
& m=0.7 \quad \sigma=\rho / \rho_{S L} \quad c_{1}<0 \quad c_{2}>0 \quad\left|c_{1}\right|>>\left|c_{2}\right|
\end{aligned}
$$

The parameter $c_{2}$ can often be neglected for the existing takeoff speed range. The momentum drag, which is airspeed dependent and opposes thrust is expressed with the linear term. The maximum takeoff (MTO) or maximum continuous (MCT) thrust flat-ratings ratings are incorporated into thrust setting parameter (TSP). Average thrust of one engine can be calculated using EAS:

$\bar{T}_{1 e}=\frac{1}{v} \int_{0}^{v} T S P \cdot T_{0} \sigma^{m}\left(1+\frac{c_{1} v}{\sqrt{\sigma}}+\frac{c_{2} v^{2}}{\sigma}\right) d v=T S P \cdot T_{0} \sigma^{m}\left(1+\frac{c_{1} v}{2 \sqrt{\sigma}}+\frac{c_{2} v^{2}}{3 \sigma}\right)$

A sudden and complete engine failure at engine failure speed $V_{\mathrm{EF}}$ and the complete loss of thrust on failed engine is assumed resulting in the average net AEO and OEI accelerations for constant mass/weight:

$$
\begin{aligned}
& \bar{a}_{A E O}=\frac{g}{W} \cdot \bar{F}_{A E O}=\frac{g}{W} \cdot\left[n_{e} \bar{T}_{1 e}+\bar{\mu}_{r} \bar{L}-\bar{D}_{a}-\left(\bar{\mu}_{r}+\bar{\phi}\right) W\right] \\
& \bar{a}_{O E I}=\frac{g}{W} \cdot \bar{F}_{O E I}=\frac{g}{W} \cdot\left[\left(n_{e}-1\right) \bar{T}_{1 e}+\bar{\mu}_{r} \bar{L}-\bar{D}_{a}-\left(\bar{\mu}_{r}+\bar{\phi}\right) W\right]
\end{aligned}
$$

All forces above are average during takeoff except that aircraft weight is assumed constant. Average acceleration during no-wind takeoff is practically equal to instantaneous acceleration evaluated at the speed equal to $70 \%$ of the liftoff speed (Durbin and Perkins, 1962; Mair and Birdsall, 1992; Saarlas, 2007). Normally, aerodynamic drag and tire resistance, while on the ground, would increase somewhat during OEI condition compared to AEO case because of the control inputs and the adverse elevator effect (AEE) during rotation (Daidzic, 2014 b), but that minor short-duration effect is being omitted from analysis. Assuming that coefficients $A, B$, and $C$ are constant in the given sped-interval, the time elapsed during portions (or entire) takeoff roll is:

$$
t=\int_{v_{1}}^{v_{2}} \frac{d v}{A+B \cdot v+C \cdot v^{2}}
$$

where (Spiegel and Liu, 1999): 


$$
\int_{v_{1}}^{v_{2}} \frac{d x}{A+B \cdot x+C \cdot x^{2}}=\frac{1}{\sqrt{Z}} \cdot \ln \left\{\left[\frac{2 C v_{2}+B-\sqrt{Z}}{2 C v_{1}+B-\sqrt{Z}}\right] \cdot\left[\frac{2 C v_{1}+B+\sqrt{Z}}{2 C v_{2}+B+\sqrt{Z}}\right]\right\}
$$

The average acceleration can be now defined and calculated from:

$$
\frac{v^{2}}{2 \bar{a}}=\int_{0}^{v} \frac{v d v}{A+B \cdot v+C \cdot v^{2}}
$$

where, for $Z=B^{2}-4 \cdot A \cdot C>0$, (Spiegel and Liu, 1999):

$$
\begin{aligned}
& \int_{v_{1}}^{v_{2}} \frac{x d x}{A+B \cdot x+C \cdot x^{2}}=\frac{1}{2 C} \ln \left[\frac{A+B v_{2}+C v_{2}^{2}}{A+B v_{1}+C v_{1}^{2}}\right]-\frac{B}{2 C} \int_{v_{1}}^{v_{2}} \frac{d x}{A+B \cdot x+C \cdot x^{2}}= \\
& =\frac{1}{2 C} \ln \left[\frac{A+B v_{2}+C v_{2}^{2}}{A+B v_{1}+C v_{1}^{2}}\right]-\frac{B}{2 C \sqrt{Z}} \ln \left\{\left[\frac{2 C v_{2}+B-\sqrt{Z}}{2 C v_{1}+B-\sqrt{Z}}\right] \cdot\left[\frac{2 C v_{1}+B+\sqrt{Z}}{2 C v_{2}+B+\sqrt{Z}}\right]\right\}=
\end{aligned}
$$

The average values of maximum rolling friction and (effective) runway slope during ground roll are:

$$
\bar{\mu}_{r}=\frac{1}{v} \int_{0}^{v} \mu_{r}(v) d v \quad \bar{\phi}=\frac{1}{v} \int_{0}^{v} \phi(v) \frac{v}{a} d v
$$




\section{Appendix $B$}

Newton-Raphson method for system of simultaneous nonlinear equations

Let us assume a vector of functions with the vector of independent values that needs to be zeroed. We are seeking vector of solutions $x_{i}$ that satisfies:

$F_{i}\left(x_{1}, x_{2} \ldots, x_{N}\right)=0 \quad i=1,2, \ldots, N$

A multi-variable functions can be expanded in Taylor series where only first-order (linear) terms are retained (Press et al., 1992):

$F_{i}(\mathbf{x}+\delta \mathbf{x})=F_{i}(\mathbf{x})+\sum_{j=1}^{N} \frac{\partial F_{i}}{\partial x_{j}} \delta x_{j}+O\left(\delta x^{2}\right)$

The matrix of partial derivatives defines, so-called, Jacobi matrix or Jacobian (e.g., Press et al., 1992) is defined as:

$J_{i j} \equiv \frac{\partial F_{i}}{\partial x_{j}}$

By neglecting the higher-order terms in the above Taylor expansion and setting $\mathbf{F}(\mathbf{x}+\delta \mathbf{x})=0$, one obtains in matrix notation:

$\mathbf{J} \cdot \delta \mathbf{x}=-\mathbf{F}(\mathbf{x})$

And accordingly iteratively:

$\mathbf{x}_{k+1}=\mathbf{x}_{k}-\mathbf{J}^{-1} \cdot \mathbf{F}(\mathbf{x})$

The Jacobian must be non-singular matrix for which determinant exists. Unfortunately, the quadratic convergence of the Newton-Raphson numerical method strongly depends on the initial guess of independent variables and is by no means assured. The global root-finding methods exist, but are much slower and quite more complex. For complicated functions or when not supplied in analytic form, the Jacobian can be estimated using numerically evaluated functions derivatives (Press et al., 1992). Many of the advanced solvers for nonlinear system of equations are implemented in IMSL for Fortran 90/95/2003. 


\section{Appendix $C$}

Finding optimum solutions for multi-variable functions

Let us assume we have a function of $n$ independent variables. We are seeking stationary points or optimums/extremums (maximum, minimum, saddle points) of this function. Some extremums may be local and some are also global. For the sake of simplicity we will use a function of two independents, which can easily be expanded to $n$ variables. We can expand such function into Taylor series around a known $z_{o}=f\left(x_{o}, y_{0}\right)$ (Ayres and Mendelson, 2009; Smith, 1953):

$$
\begin{aligned}
& f\left(x_{o}+h, y_{0}+k\right)=f\left(x_{o}, y_{0}\right)+d f\left(x_{o}, y_{0}\right)+\frac{1}{2} d^{2} f\left(x_{o}+\theta h, y_{0}+\theta k\right)= \\
& =f\left(x_{o}, y_{0}\right)+\left[\frac{\partial f}{\partial x} h+\frac{\partial f}{\partial y} k\right]_{\left(x_{o}, y_{0}\right)}+\frac{1}{2}\left[\frac{\partial^{2} f}{\partial x^{2}} h^{2}+2 \frac{\partial^{2} f}{\partial x \partial y} h k+\frac{\partial^{2} f}{\partial y^{2}} k^{2}\right]_{(x, y)}+\ldots \\
& x=x_{0}+\theta h \quad y=y_{0}+\theta k
\end{aligned}
$$

We can now define elements of the square Hessian matrix (matrix of second-order partial derivatives) around a known point $P$ :

$$
\left.[H]_{i, j} \equiv \frac{\partial^{2} f}{\partial x_{i} \partial x_{j}}\right|_{P}
$$

Trace of the non-singular Hessian matrix of rank $n$ is:

$$
\operatorname{tr}[H]=\sum_{i=1}^{n} \frac{\partial^{2} f}{\partial x_{i}^{2}}
$$

For two independent variables (Equation C1), the determinant of the nonsingular Hessian matrix is simply:

$$
|H|=\left|\begin{array}{ll}
\frac{\partial^{2} f}{\partial x^{2}} & \frac{\partial^{2} f}{\partial x \partial y} \\
\frac{\partial^{2} f}{\partial y \partial x} & \frac{\partial^{2} f}{\partial y^{2}}
\end{array}\right|=\frac{\partial^{2} f}{\partial x^{2}} \cdot \frac{\partial^{2} f}{\partial y^{2}}-\left(\frac{\partial^{2} f}{\partial x \partial y}\right)^{2} \neq 0 \quad \frac{\partial^{2} f}{\partial x \partial y}=\frac{\partial^{2} f}{\partial y \partial x}
$$


The necessary condition for the multivariable function to have extremum at point $P$ is that all first derivative vanish (Ayres and Mendelson, 2009; Smith, 1953):

$\left.\frac{\partial f}{\partial x_{i}}\right|_{P}=0$

Depending on the determinant of the nonsingular Hessian matrix (Ayres and Mendelson, 2009; Chapra and Canale, 2006; Smith, 1953), we can define maximum, minimum, or the saddle point at established extremum point $P$ for the function of two independent variables as:

$$
|H| \Rightarrow\left\{\begin{array}{ccc}
>0 & \frac{\partial^{2} f}{\partial x_{i}^{2}}<0 & \text { maximum } \\
<0 & \frac{\partial^{2} f}{\partial x_{i}^{2}} \cdot \frac{\partial^{2} f}{\partial x_{j}^{2}}<0 & \text { saddle point } \\
>0 & \frac{\partial^{2} f}{\partial x_{i}^{2}}>0 & \text { minimum }
\end{array}\right\}
$$




\section{Appendix D}

Density altitude calculations

Density altitude (DA) calculations for standard and nonstandard temperatures and constant pressure altitudes (PA) are illustrated in Figure D1.

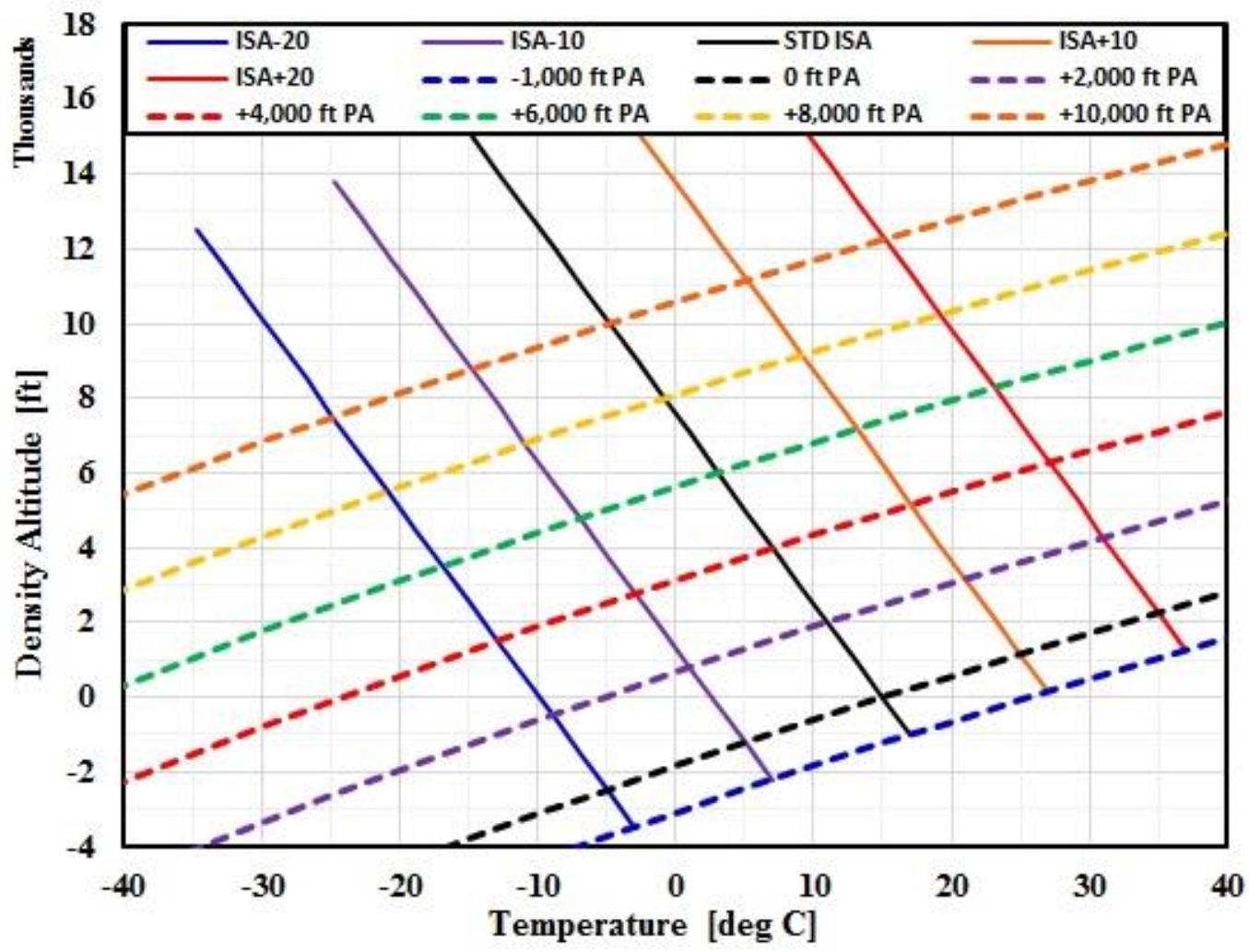

Figure D1. Variations of DA for standard and nonstandard ISA temperature conditions and various PAs.

The lines of constant PA (dashed) are also lines of constant $\delta$. The lines of constant temperature ISA $\pm \Delta \mathrm{T}$ (solid) are lines of constant $\theta$ and the lines of constant DA (horizontal) are the lines of constant $\sigma$.

Pressure altitude for ISA troposphere is (Daidzic, 2015a):

$$
P A=1.47213619 \times 10^{5} \cdot\left(1-\delta^{0.1902632}\right) \quad[\mathrm{ft}]
$$


Density altitude for ISA troposphere is (Daidzic, 2015a)

$$
D A=1.47213619 \times 10^{5} \cdot\left(1-\sigma^{0.2349692}\right) \quad[\mathrm{ft}]
$$

